ARTICLE

DOI: $10.1038 / s 41467-018-04184-x$

\title{
Hox and Wnt pattern the primary body axis of an anthozoan cnidarian before gastrulation
}

Timothy Q. DuBuc ${ }^{1}$, Thomas B. Stephenson², Amber Q. Rock² \& Mark Q. Martindale (D) ${ }^{2}$

Hox gene transcription factors are important regulators of positional identity along the anterior-posterior axis in bilaterian animals. Cnidarians (e.g., sea anemones, corals, and hydroids) are the sister group to the Bilateria and possess genes related to both anterior and central/posterior class Hox genes. Here we report a previously unrecognized domain of Hox expression in the starlet sea anemone, Nematostella vectensis, beginning at early blastula stages. We explore the relationship of two opposing Hox genes (NvAx6/NvAx1) expressed on each side of the blastula during early development. Functional perturbation reveals that NvAx6 and NvAx1 not only regulate their respective expression domains, but also interact with Wnt genes to pattern the entire oral-aboral axis. These findings suggest an ancient link between Hox/Wnt patterning during axis formation and indicate that oral-aboral domains are likely established during blastula formation in anthozoan cnidarians.

\footnotetext{
${ }^{1}$ Centre for Chromosome Biology, Bioscience Building, National University of Ireland Galway, Galway H91 RTR6, Ireland. ${ }^{2}$ Whitney Lab for Marine Bioscience and the Department of Biology, University of Florida, St. Augustine, FL 32080, USA. These authors contributed equally: Timothy Q. DuBuc, Thomas B. Stephenson. Correspondence and requests for materials should be addressed to M.Q.M. (email: mamartin@whitney.ufl.edu)
} 
$o x$ genes are a specific family of homeobox-containing transcription factors that have been studied extensively in several clades of bilaterally symmetrical animals ("bilaterians", Fig. 1a). Originally discovered in Drosophila ${ }^{1}$, Hox genes play an important role in establishing segment identity along the anterior-posterior (A-P) axis during development and are conserved in all bilaterian lineages including vertebrates ${ }^{2}$. Hox genes are often clustered together along contiguous stretches of an animal's genome $\mathrm{e}^{3}$, and are classified into three paralogy groups: anterior (Hox1-3), central (Hox4-8), and posterior (Hox9-13) (Fig. 1b). Early comparisons of vertebrate and insect Hox sequences revealed that homologous Hox genes occupy similar locations in the $3^{\prime}-5^{\prime}$ topology of the cluster ${ }^{4}$ and gene expression analysis further revealed that a gene's position in the cluster correlated with its expression profile along the A-P axis. Although there are derivations in the organization and distribution of Hox genes among bilaterians, Hox genes are consistently expressed in anterior-to-posterior territories as reflected by their phylogenic ancestry ${ }^{5-11}$. Furthermore, duplications (vertebrates) and fragmentation of Hox clusters (invertebrates) likely had a strong impact on the radiation of animals ${ }^{8,12}$, although the origin and function of this gene family within the first invertebrates is less clear ${ }^{13-16}$.

Phylogenetic reconstructions of the bilaterian Hox gene compliment indicate that the common ancestor of most extant animals had a Hox cluster containing anterior, central, and posterior Hox genes ${ }^{17,18}$ (Fig. 1b, c). Although clustering is not an essential component of Hox functionality, it is widely associated with diverse animal clades from cnidarians and throughout Bilateria. Cnidarians (e.g., corals, anemones, and hydroids) are the sister group to the Bilateria ${ }^{19}$ (Fig. 1a) and possess bona fide Hox genes related to anterior and central/posterior Hox genes ${ }^{14,15,20-23}$. The cnidarian common ancestor likely had a Hox cluster consisting of at least two types of Hox genes, related to anterior and central/ posterior $^{21,22}$ (Fig. 1d, Supplementary Fig. 1). Definitive central class genes have yet to be found in a cnidarian. They first appear in the Xenoacoelomorph bilaterian lineage ${ }^{24}$ and likely expanded in protostome/deuterostome lineages (Fig. 1b, c, Supplementary Fig. 1). Currently, it is uncertain whether central class Hox genes arose in the bilaterian lineage from an ancestral central/posterior Hox gene, or were lost in the cnidarian lineage. Bona fide Hox genes have yet to be found in the genomes of earlier branching animal clades ${ }^{25-28}$, although some evidence suggests gene loss may have impacted this gene family ${ }^{16}$ and a greater effort needs to be made to survey the diverse group of extant cnidarians. Efforts to functionally characterize the cnidarian Hox complement are crucial for determining the pre-bilaterian role of these important developmental regulators.

Functional studies in the fly ${ }^{1}$ and mouse ${ }^{29}$ first showed that Hox genes are causally involved in establishing adult body structures from the region in which they are expressed, thus controlling regional identity along the A-P axis. Hox genes also interact indirectly with one another in overlapping domains, with posterior Hox genes having functional "dominance" over more anterior genes; however, flies also exhibit examples of anterior dominance ${ }^{30}$. mRNA expression studies of Hox genes in multiple cnidarian species suggest a discrete role in late larval patterning, yet the developmental function remains untested $13,15,20,21,31,32$. The Hox cluster of the starlet sea anemone Nematostella consists of two neighboring non-Hox homeobox genes ( $N v H l x B 9$ and $N v E v e$ ), along with putative orthologs to the anterior Hox genes Hox $1(N v A x 6)$ and Hox $2(N v A x 7 \text { and } N v A x 8)^{14,15}$. Four additional Hox genes exist in the genome, consisting of: two central/ posterior genes $(N v A x 1$ and $N v A x 1 a)$, a pseudogene $(N v A x 9)$, and an additional anterior-like gene of indeterminate orthology $(N v A x 6 a)$ (Fig. 1e). The assemblage of Hox clusters from different cnidarian genomes indicate that anthozoan cnidarians had a relatively intact Hox cluster, which has been fragmented in different species (Supplementary Fig. 1). Corals arguably have the most complete cluster consisting of both anterior and central/ posterior orthologs ${ }^{22}$ (Supplementary Fig. 1c). Representative anemone species (Nematostella and Aiptasia) appear to have diverged from a cluster seen in some corals with a duplicated Hox 2 ortholog $(A x 7$ and $A x 8)$ (Supplementary Fig. 1d-e). An ortholog of $N v A \times 1$ is present in all cnidarian genomes, yet there is no evidence of it being linked to a cnidarian cluster, suggesting it diverged long ago. In the case of Hydra, a high rate of cluster fragmentation is correlated with an expansion of central/posterior genes and less conservation among anterior-like genes ${ }^{14,15}$ (Supplementary Fig. 1f). Upcoming genome projects of other Hydrozoan cnidarians, Clytia hemisphaerica and two Hydractinia species, may reveal more details about the distribution of these genes. All of these findings are based on the idea that the ancestral condition was a state of Hox clustering, which is generally assumed to be the representative condition among bilaterian clades. A lack of functional data only allows us to speculate on the impact of cluster fragmentation within Cnidaria, and additional work is needed to resolve the impact of the cnidarian Hox diversification.

Previous in situ hybridization studies conducted on larval and juvenile stages of Nematostella revealed that the majority of $N v H o x$ genes are expressed in a staggered domain along the primary (oral-aboral) axis ${ }^{15}$ with the anterior Hox gene (NvAx6) expressed in a region corresponding to the pharyngeal nerve ring at the oral pole, and the central/posterior Hox gene $(N v A x 1)$ expressed in the larval apical tuft at the aboral tip of the planula (Fig. 1e). The rest of the N. vectensis Hox genes are expressed later in development at planula stages asymmetrically along one side of the endoderm within the oral-aboral domain separating $N v A x 6$ and $N v A x 1^{15,20}$. These data along with other studies in medusazoan cnidarians that possess complex metagenic lifecycles have created uncertainty as to whether cnidarian Hox genes have any bilaterian-like attributes. Early work using the cnidarian model system, Hydra, found a role in adult oral-aboral patterning $31,33,34$, while more recent findings concluded that the axial patterning role of Hox genes arose after the cnidarian bilaterian split ${ }^{13,21,32}$. Here we begin to dissect for the first time the functional role and hierarchy of Hox genes during cnidarian embryonic development and generate a presumptive molecular network for oral-aboral patterning.

\section{Results}

Hox genes are expressed prior to germ layer segregation. We analyzed the expression of Hox and neighboring non-Hox homeobox genes in Nematostella throughout early development (Fig. 2a), using a highly sensitive method (quantitative PCR) and verified expression by in situ hybridization. mRNA of both anterior and central/posterior Hox genes were detected early in development (Fig. 2b). Maternal mRNA of $N v A x 1$ and $N v A x 6 a$ could be detected by qPCR in fertilized eggs (Fig. 2b, 0hpf); however, transcriptional activation of the extended Hox gene cluster begins during early blastula formation (Fig. 2b, 12hpf) with expression of the anterior Hox gene $N v A x 6$. Deployment of the Hox cluster continues during the blastula to gastrula transition, with the activation of $N v E v e$ (Fig. 2b, 16hpf) and two genes related to Hox2 ( $N v A x 7$ and $N v A x 8$ ) (Fig. 2b, 18hpf). Lastly, activation of the central/posterior Hox gene $(N v A x 1 a)$, which is fragmented from the predicted ancestral cnidarian Hox gene cluster (Fig. 1d) ${ }^{22}$, begins near the onset of gastrulation (Fig. 2b, 24hpf). 

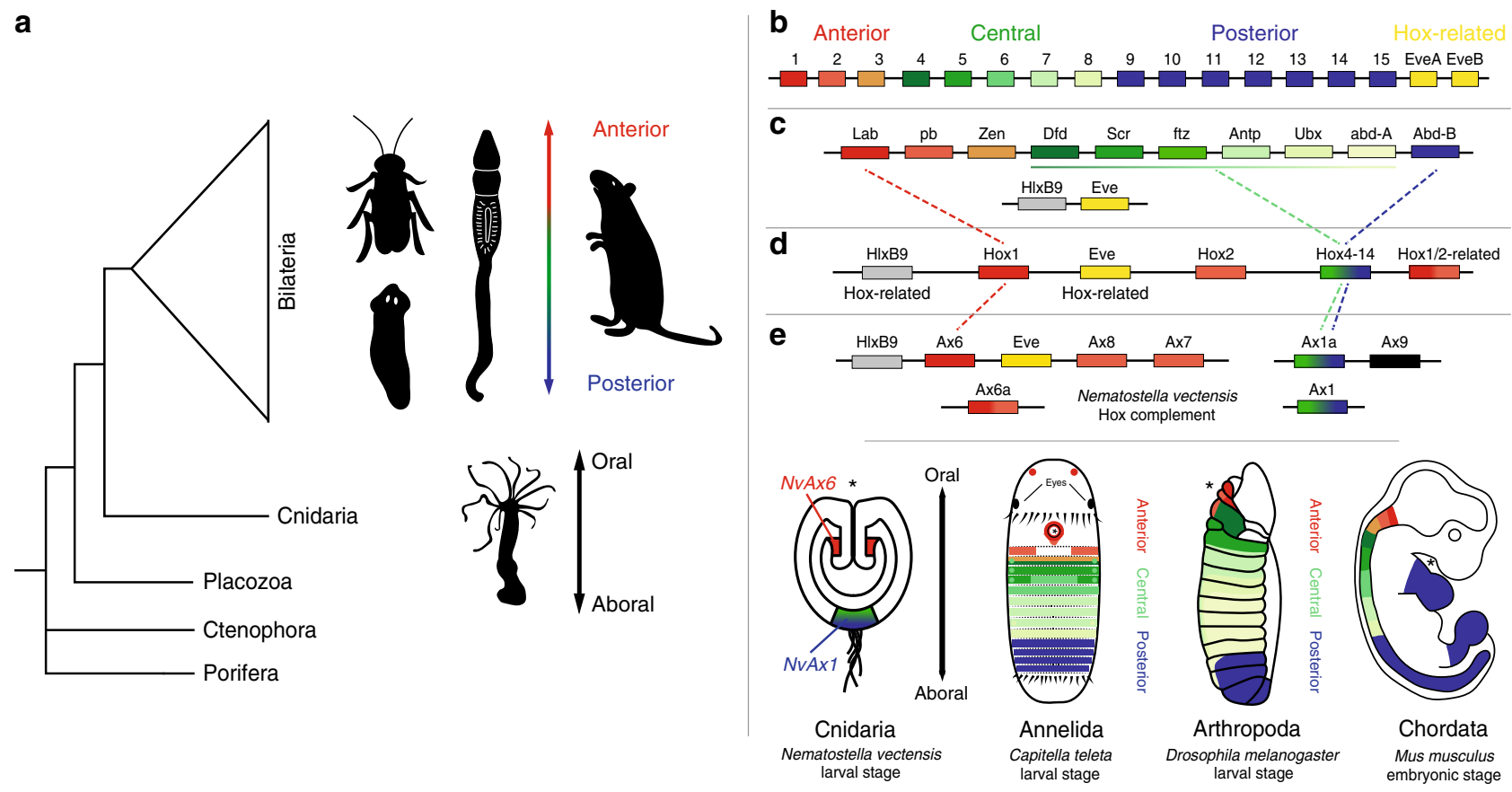

Fig. 1 Anterior-posterior patterning and the emergence of a Hox cluster. a Bilaterians are classically defined by an anterior-posterior axis perpendicular to the dorsal ventral axis. Cnidarians are the sister taxa to bilaterians and are the only basal lineage to have a diverse cluster of Hox genes. $\mathbf{b}$ The common ancestor of the deuterostome lineage likely had a Hox cluster consisting of 14-15 Hox genes, closely associated with the homeobox gene Eve ${ }^{18}$. $\mathbf{c}$ Evidence from the protostome, Tribolium castaneum, suggests that the protostome ancestor also had an intact Hox cluster consisting of at least 10 linked Hox genes ${ }^{17,70}$. d The cnidarian ancestor had both anterior (Hox1 and Hox2) and central/posterior (Hox9-13) class Hox genes ${ }^{22}$. e The Hox complement of the anthozoan cnidarian, Nematostella vectensis, has phylogenetically anterior ( $N v A x 6, N v A x 6 a, N v A x 7$, and NvAx8) and central/posterior (NvAx1 and NvAx1a) Hox genes ${ }^{14,15}$. Depiction of Hox expression along the oral-aboral axis of a cnidarian, and the anterior-posterior axis of invertebrates and vertebrates. The anterior (NvAx6) and central/posterior (NvAx1) Hox genes of Nematostella are expressed along the oral-aboral axis during larval development. Regions of anterior, central, and posterior Hox expression are designated with shades of red, green, and blue, respectively. Asterisk indicates site of mouth formation

Two zones of spatial activation were found, with genes being expressed in oral $(N v A x 6, N v A x 6 a, N v E v e, N v A x 7$,$) or aboral$ $(N v A x 1, N v A x 8)$ domains before and during early gastrulation prior to any asymmetries along the directive axis (Fig. 2b). Notably, $N v A x 6$ and $N v A x 1$ were the first to be detected by in situ hybridization, occupying complimentary oral and aboral domains during early blastula formation (12hpf) (Fig. 2c). $N v H l x B 9$ and $N v E v e$ are expressed along the oral pole of the animal during early gastrulation $^{35}$, and in agreement with the GPCR analysis (Fig. 2b), the $N v H o x$ genes appear to be expressed along a similar temporal timeline. Respective oral and aboral expression of $N v A x 6$ and $N v A x 1$ continues through the blastula to gastrula transition. At this stage $N v A x 6$ is initially expressed at the site of gastrulation (animal pole) in the presumptive endomesoderm before becoming restricted to the pharyngeal endoderm (Fig. 3a-e) associated with the pharyngeal nerve ring ${ }^{15,20}$. Conversely, $N v A x 1$ maintains a broad aboral expression domain throughout blastula and gastrula stages before becoming refined to the most aboral domain of the apical tuft region during early planula stages (Fig. 3f-j). The broad complimentary expression domains of the orally expressed anterior Hox gene $N v A x 6$ and aborally expressed $N v A x 1$ genes during blastula stages suggests they may play an important role in oral-aboral patterning during early development.

Unequal opposing role of Hox genes in oral-aboral patterning. $N v A x 6$ and $N v A x 1$ are expressed in opposite territories throughout development and their function was manipulated by microinjection of mRNA or antisense oligonucleotides into uncleaved zygotes. Knockdown of the orally expressed anterior
Hox gene, NvAx6, results in embryos that have defective gastrulation, compared with controls (Fig. 3k), and only initially form an inner endomesodermal plate (Fig. 31). Overexpression of the aborally expressed Hox gene, $N v A x 1$, by mRNA microinjection, results in a loss of gastrulation (Fig. $3 \mathrm{~m}$ ). Overexpression of the anterior Hox gene $N v A x 6$ or morpholino knockdown of $N v A x 1$, each interfere with pharyngeal development and produce external tissue with a pronounced asymmetry at the blastopore that fails to invaginate (Fig. 3n, o). At early gastrula stages (24hpf), knockdown of the orally expressed Hox gene, $N v A x 6$, and the aborally expressed Hox gene, $N v A x 1$, caused an expansion of $N x A x 1$ and $N v A x 6$ expression respectively (Fig. $3 \mathrm{p}-\mathrm{q}, \mathrm{s}, \mathrm{t}$ ). However, overexpression of the anterior Hox gene NvAx6 exhibits only a slight expansion of $N v A x 1$ expression (Fig. $3 \mathrm{u}$ ). Conversely, overexpression of aboral Hox gene, $N v A x 1$, completely abolishes $N v A x 6$ expression at the oral pole (Fig. $3 \mathrm{r}$ ) and inhibits oral development (Fig. $3 \mathrm{~m}$ ). This suggests that $N v A x 6$ and $N v A x 1$ maintain respective oral and aboral expression domains through antagonism, but that $N v A x 1$ appears to have functional dominance over $N v A x 6$, or that other factors contribute to the lack of antagonism in $N v A x 6$ overexpression experiments (Fig. $3 \mathrm{u}$ ).

The morphological changes found during early development and gastrulation are consistent with phenotypes identified during larval and polyp formation (Fig. 4). Three days after injection, ectopic tissue around the larval mouth is still present in animals with overexpression of $N v A x 6$, where overexpression of $N v A x 1$ inhibits larval development (Fig. $4 \mathrm{a}-\mathrm{c}$ ). The aboral sensory apical tuft is only affected by overexpression or knockdown of $N v A x 1$ (Fig. $4 \mathrm{~d}-\mathrm{h}$ ). Finally, overexpression of $N v A x 6$ results in ectopic tissue also in the juvenile polyp, consistent with expansion of oral territories created during gastrulation. $N v A x 1$ overexpression 
a

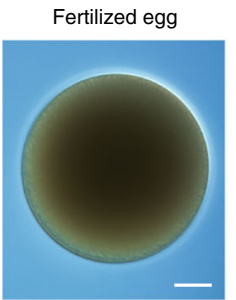

$0 \mathrm{hpf}$

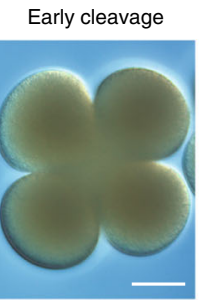

$2 \mathrm{hpf}$

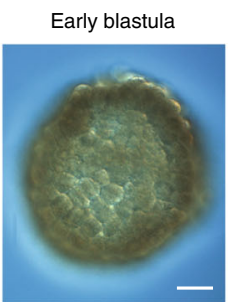

$8 \mathrm{hpf}$

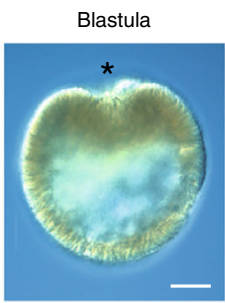

$24 \mathrm{hpf}$
Planula larva

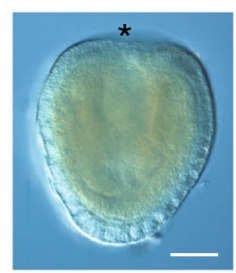

3 days old

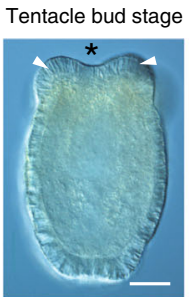

5 days old

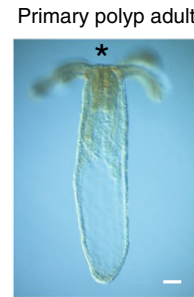

7-10 days old b

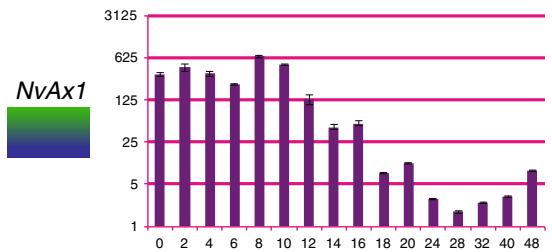

NvAx6
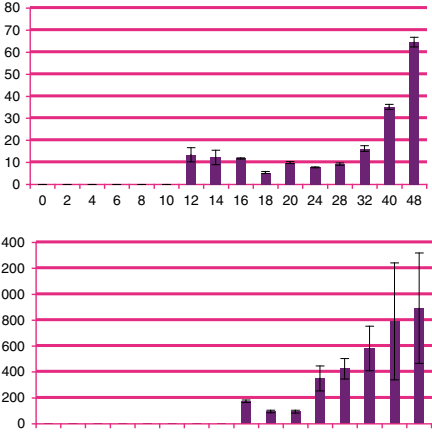

NvAx8

NVAX8

NvAx 7

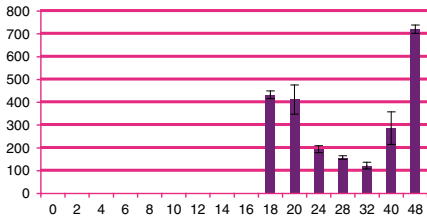

4000

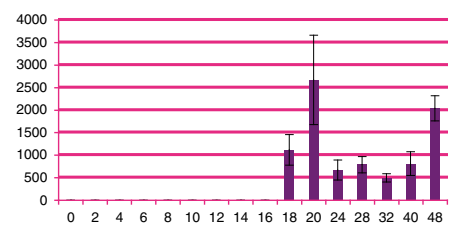

NvAx1a

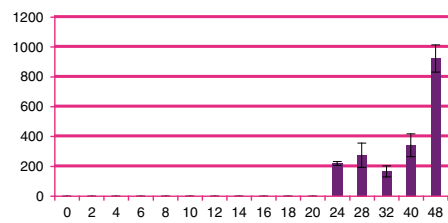

NvAx6a

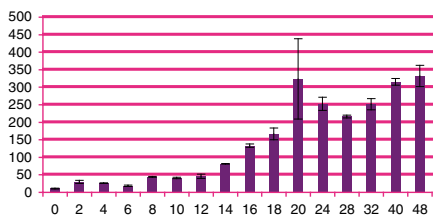

Hours post fertilization (hpf)

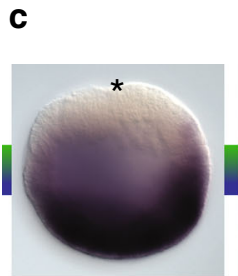

Spatial mRNA expression domain (in situ hybridization)
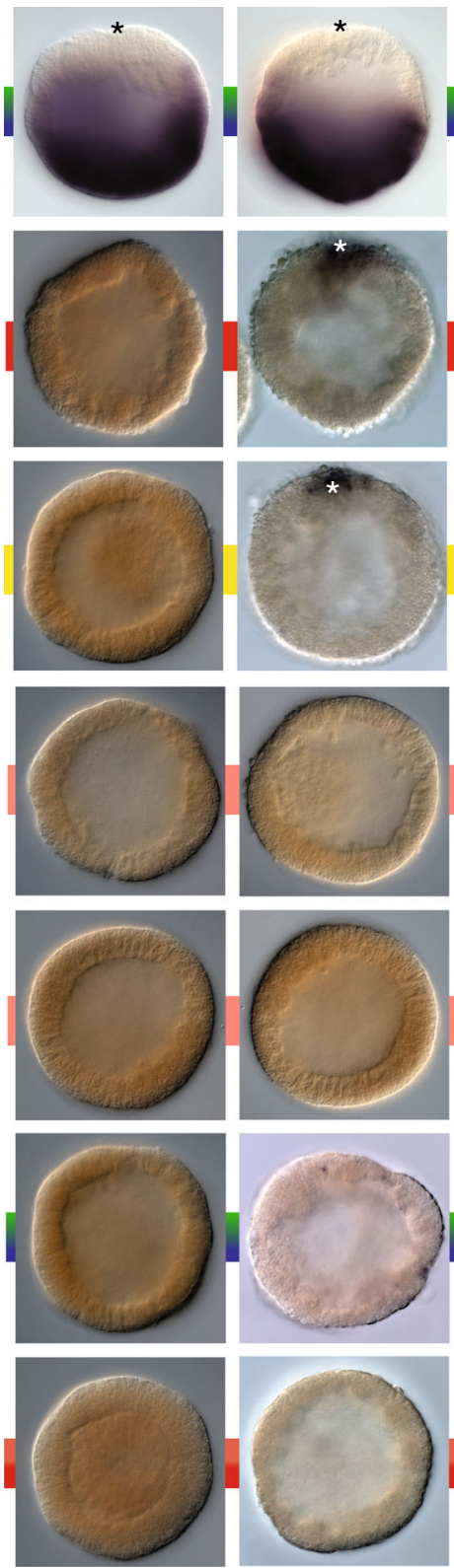

$10 \mathrm{hpf}$
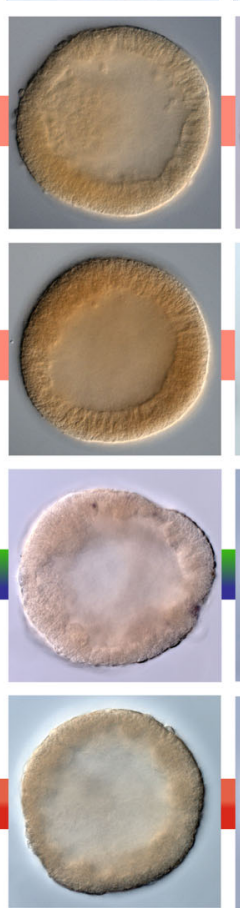

12 hpf
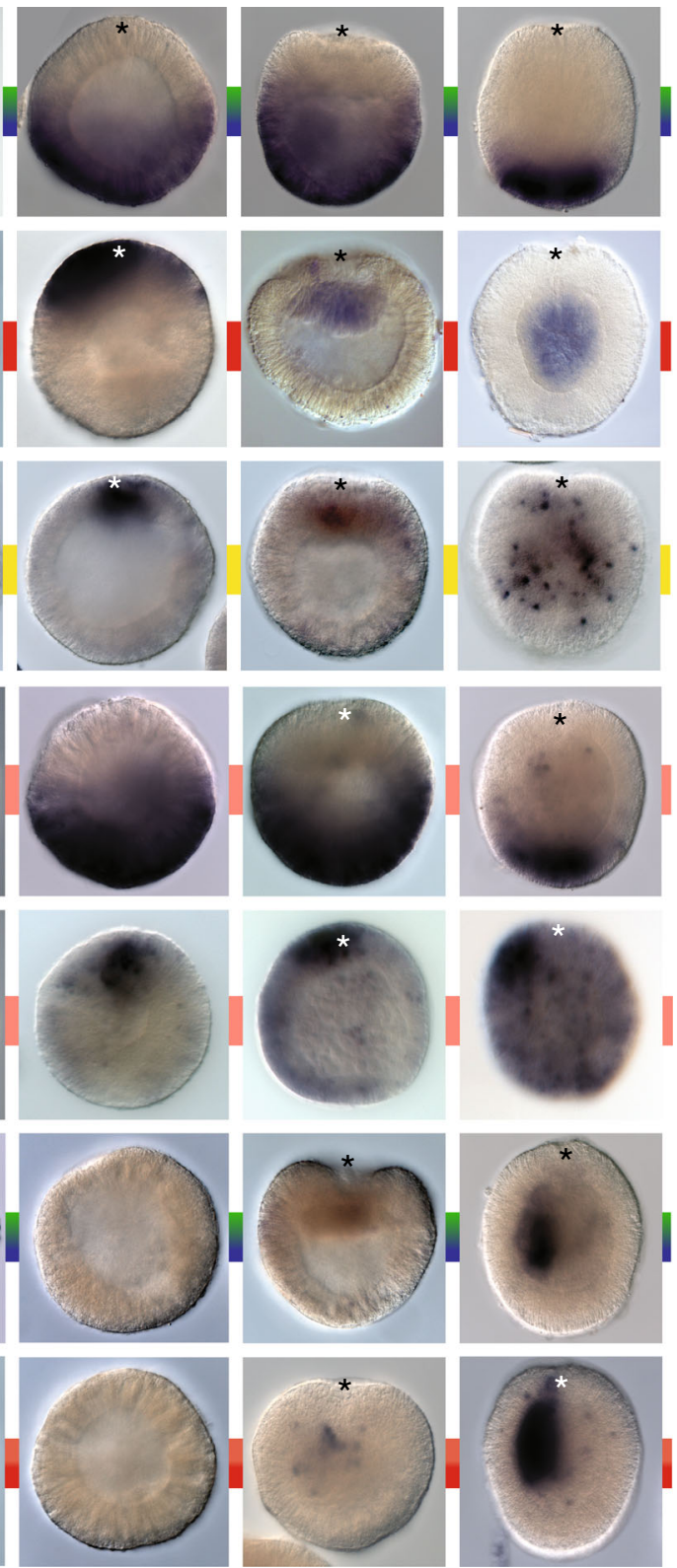

18 hpf

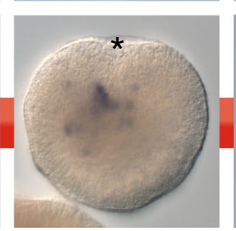

$24 \mathrm{hpf}$

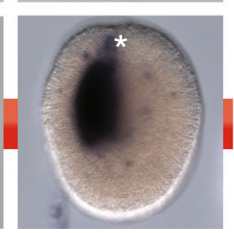

$48 \mathrm{hpf}$

Fig. 2 Hox genes of Nematostella exhibit hallmarks of bilaterian Hox genes during early development. a Time series of the embryonic, larval, and adult development of the cnidarian, Nematostella vectensis (hours post fertilization =hpf). b Clustered Hox genes display temporal colinearity during early embryonic development. Expression begins with the 3' located neighboring non-Hox homeobox gene NvHlxB9 (data not shown) and the anterior gene NvAx6 at $12 \mathrm{hpf}$. Subsequent activation of the other genes in the cluster maintains colinear expression relative to the ancestral cnidarian cluster. Two of the unlinked Hox genes NVAx 1 (central/posterior) and NvAx6a (anterior) appear maternally expressed during early development. $\mathbf{c}$ In situ hybridization confirms the temporal activation of the Hox cluster. Prior to gastrulation, mRNA expression of the central/posterior Hox gene (NvAxi) localizes to the aboral pole, while an anterior Hox gene (NvAx6) becomes transcriptionally activated along the oral pole. Remaining Hox and homeobox genes occupy oral (NvEve, NvAv7, NvAx6a) or aboral (NvAx8) domains during early development. Images were compiled from at least three separate experiments. Error bars are based on S.E.M. Scale bars are $50 \mu \mathrm{m}$ 

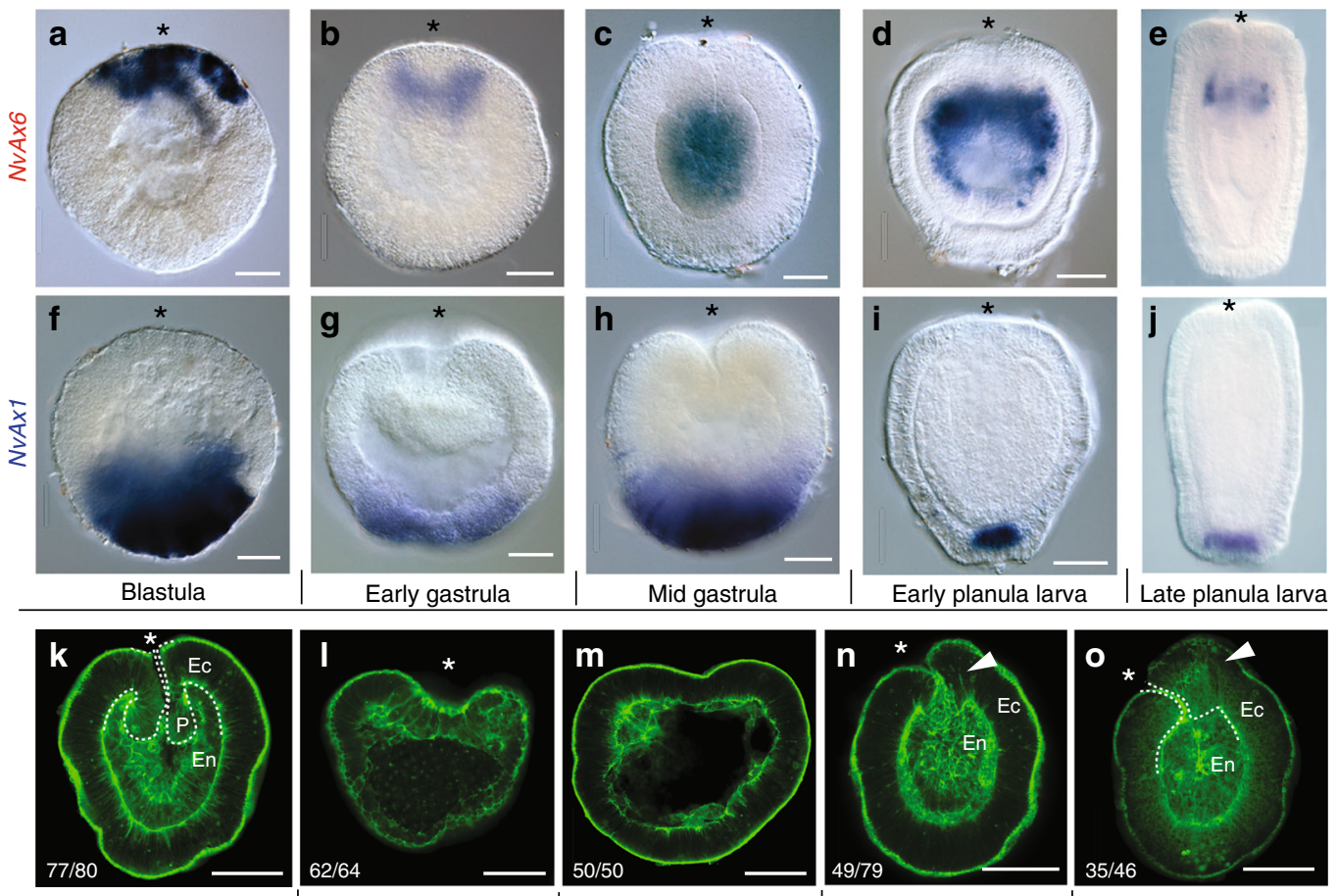

Control gastrula (48 hpf)

NvAx6 knockdown

NvAx1 overexpression

NvAx6 overexpression

NvAx1 knockdown
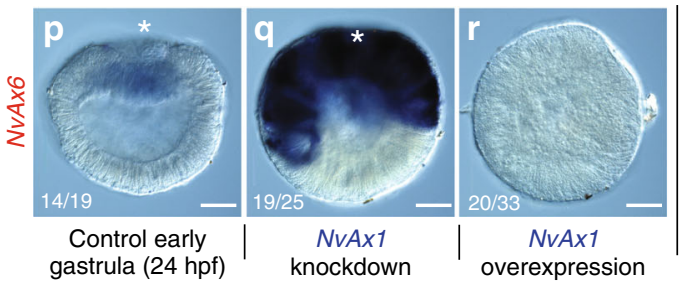

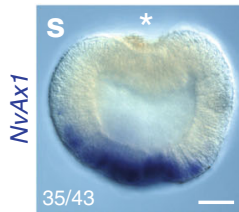

Control early gastrula (24 hpf)

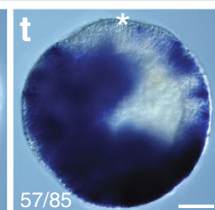

NvAx6 knockdown

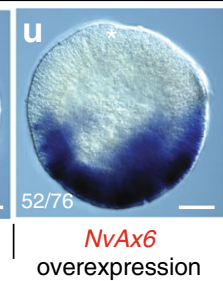

Fig. 3 Perturbation of Hox expression disrupts oral patterning during gastrulation. Developmental time series of anterior, NvAx6 (a-e), and central/ posterior, $\operatorname{NvAx1}(\mathbf{f}-\mathbf{j})$, Hox mRNA expression at blastula $(\mathbf{a}, \mathbf{f})$, early gastrula $(\mathbf{b}, \mathbf{g})$, mid gastrula $(\mathbf{c}, \mathbf{h})$, early planula (d, i), and late planula stages (e, j; images reproduced from ref ${ }^{15}$.). k-o Gastrulation defects due to disruption of anterior (NvAx6) and central/posterior (NvAx1) Hox expression through microinjection of antisense translation blocking morpholinos (knockdown) or in vitro transcribed mRNA (overexpression). k Fluorescent phalloidin-labeled embryo during final stages of gastrulation (48hpf) with distinct ectodermal(Ec) and endo-mesodermal(En) tissue layers delineated by the early pharynx (P) (white-dashed line delineates the pharynx from endomesoderm). I Knockdown of anterior Hox (NvAx6) blocks invagination of the presumptive endomesoderm. $\mathbf{m}$ Overexpression of central/posterior Hox (NvAx1) mRNA also blocks gastrulation and produces gastrula stage embryos with reduced axial morphology. $\mathbf{n}$ Overexpression of anterior Hox (NvAx6) mRNA and o knockdown of the central/posterior Hox gene (NvAx1) disrupts pharynx development and produces ectopic oral tissue at the blastopore (white arrowhead). p-r In situ hybridization of anterior Hox (NvAx6) at 24hpf in control (p), central/posterior Hox knockdown (q), and central/posterior Hox overexpression treatments (r). s-u In situ hybridization of central/posterior Hox (NvAx1) at $24 \mathrm{hpf}$ in control (s), anterior Hox knockdown (t), and anterior Hox overexpression treatments (u). Images were compiled from at least three separate experiments and the number of similar phenotypes is noted as a fraction in the lower left-hand corner (e.g., $\mathbf{k}-77$ out of 80 total animals exhibited the phenotype). Scale bars are $50 \mu \mathrm{m}$

blocks polyp formation (Fig. 4k), where knockdown of $N v A x 1$ results in deformed polyps with elongated body columns with less oral features, such as tentacles (Fig. 41).

These findings suggest that altering the Hox program during early development results in changes along the oral-aboral axis that are maintained throughout development. To understand what changes occur as a result of manipulation of Hox expression, we analyzed a diverse set of developmental patterning genes and their relationship to $N v A x 6$ and $N v A x 1$ during early development. Control injections of dextran or control morpholinos do not impair expression of any genes analyzed herein (Supplementary Fig. 2). The endomesodermal marker, NvSnailA, is reduced in $N v A x 6$ knockdown experiments and is lost in $N v A x 1$ overexpression treatments (Fig. 5a, c-e). In these two treatments, embryos continue to gastrulate (Fig. 3l-m), fail to form the pharynx, and do not express the pharyngeal marker NvFoxA or the oral marker NvBrachyury that is expressed at the ectodermal/ pharyngeal boundary (Fig. 5a), and which has been shown to regulate pharynx formation in anthozoans ${ }^{36,37}$. NvAx6 knockdown shows minor expansion of aboral markers such as $N v F G F 2 A, N v S f r p 1 / 5, N v D k k 1 / 2 / 4$, and $N v S i x 3 / 6$ (Fig. 5b), but does not appear to effect aboral development, as embryos treated with NvAx6 anti-sense morpholino eventually form a swimming planula larva with an apical tuft at the aboral pole (Fig. 4e). Surprisingly, $N v A x 1$ overexpression reduces aboral markers expression (Fig. $5 \mathrm{~b}-\mathrm{c}, \mathrm{e}$ ) and the apical tuft in this region does not form (Fig. 4f). NvSix3/6 appears both diminished and disorganized, expanding into multiple regions of the embryo (Fig. 5b-c, e).

Overexpression of the anterior Hox gene $N v A x 6$ or morpholino knockdown of $N v A x 1$ produce ectopic tissue that expresses the pharyngeal/oral markers NvFoxA and NvBrachyury (Fig. 5a, c, f, g). NvSnailA is expressed normally in cells of the endomesodermal plate indicating that these defects are related to 

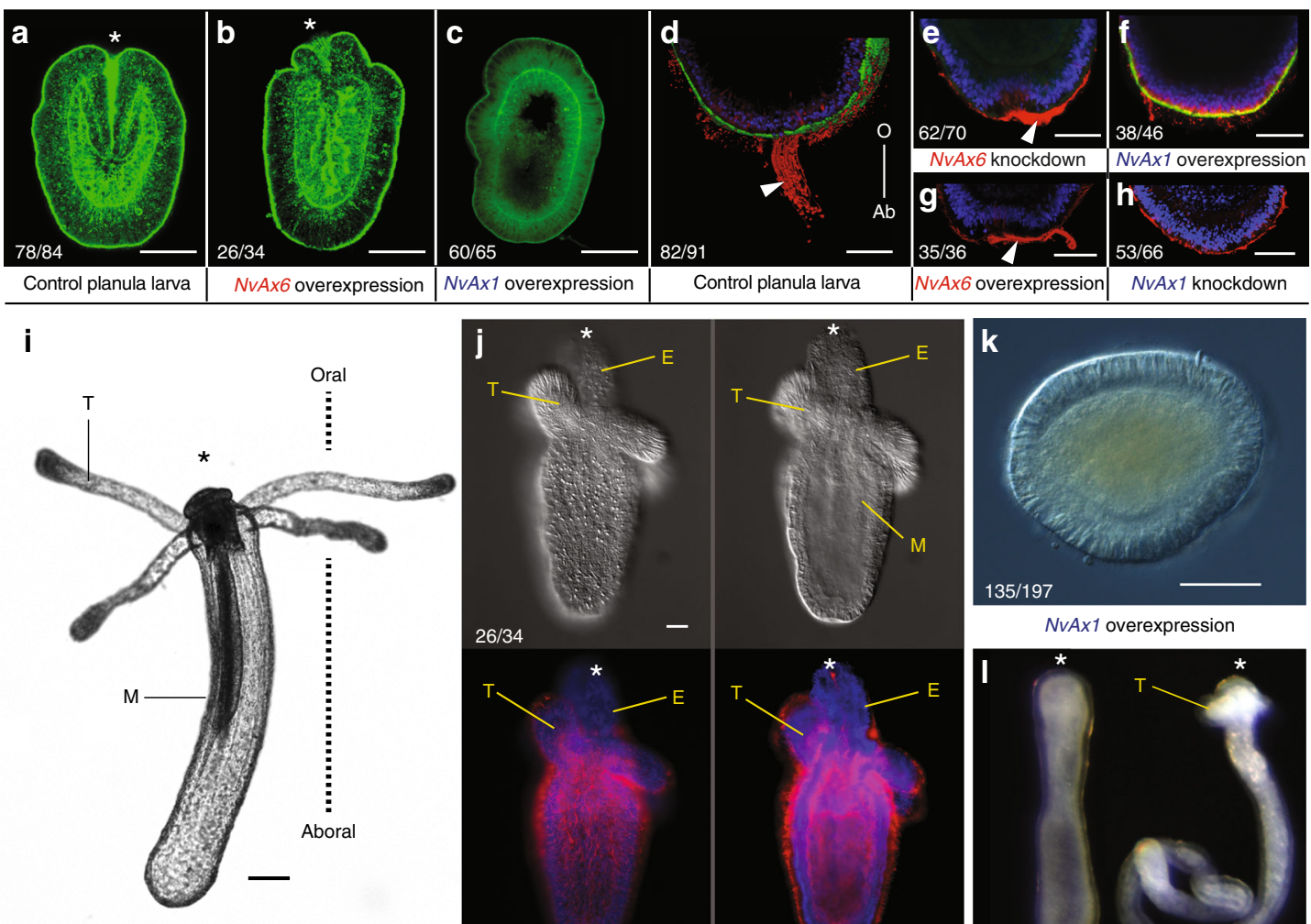

$226 / 249$
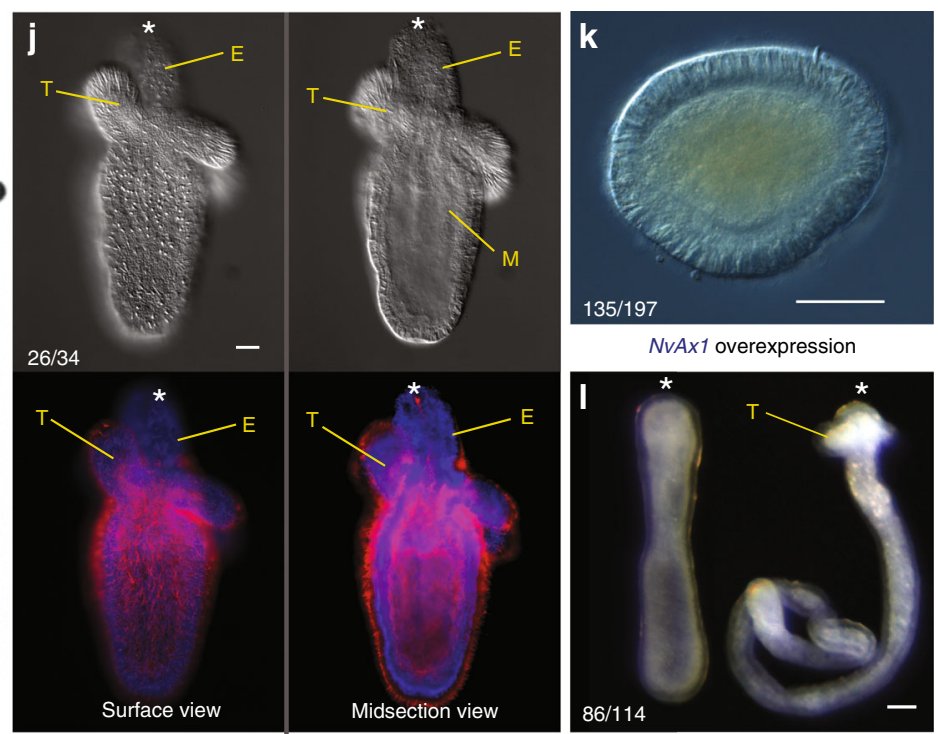

NvAx1 overexpression

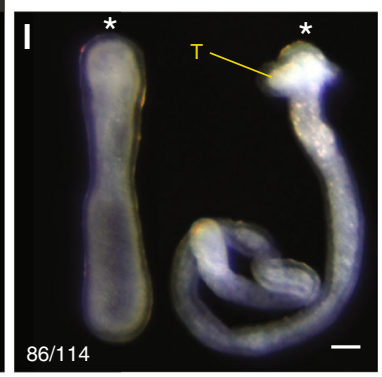

Control juvenile adult stage

NvAx6 overexpression

NvAx1 knockdown

Fig. 4 Effects of perturbing anterior and central/posterior Hox genes on oral-aboral identity extends to late development. a-c Phalloidin stained embryos at planula larvae (144hpf) stages taken from: a dextran-injected controls, b anterior Hox (NvAx6) overexpression, and c central/posterior Hox (NvAx1) overexpression treatments. $\mathbf{d}-\mathbf{h}$ Apical tuft cilia labeled with and acetylated tubulin antibody (red) with the nuclei counter stained with DAPI (blue) in: $\mathbf{d}$ planula stage embryos treated with dextran, e anterior NvAx6 morpholino, $\mathbf{f}$ central/posterior NvAx1 Hox mRNA, $\mathbf{g}$ anterior NvAx6 mRNA, or h central/ posterior NvAx1 Hox morpholino. i-I Changes in polyp morphology resulting from disruption of anterior Hox (NvAx6) and central/posterior Hox (NvAx1) expression during early development. i Wild-type polyp after metamorphosis. j Overexpression of anterior Hox (NvAx6) results in ectopic tissue formation at the oral side of the animal. $\mathbf{k}$ Overexpression of central/posterior Hox (NvAx1) results in bilayered animals (maintained for 21 days after fertilization) without clear axial morphology but have cnidocytes (stinging cells) along the outer ectoderm. I Animals treated with central/posterior Hox (NvAx1) morpholino eventually metamorphose ( 2 weeks after fertilization) to produce animals with small heads and extremely long body columns. Images were compiled from at least three separate experiments and the number of similar phenotypes is noted as a fraction in the lower left-hand corner. Scale bars are $50 \mu \mathrm{m}$

pharyngeal patterning and not endomesoderml plate specification (Fig. 5a). Aboral markers are lost in NvAx1 knockdown treatments, suggesting that the $N v A x 1$ expression is necessary for aboral specification (Fig. 5b-c, g). However, aboral marker expression and apical tuft formation are not disturbed by $N v A x 6$ overexpression (Figs 4g, 5b, c, f).

Role of $N v A x 6$ and $N v A x 1$ patterning during early development. Disruption of oral or aboral Hox genes expand opposing territories (Fig. 3q, t). Ectopic aboral Hox (NvAx1) expression restricts gastrulation and oral specification, resulting in the lack of endomesoderm formation throughout development (Fig. 5a). Similarly, knockdown of the oral Hox gene $N v A x 6$ produces a severe defect in gastrulation (Fig. 31) and results in an expansion of $N v A \times 1$ expression toward the oral pole (Fig. $3 \mathrm{t}$ ). This suggests that stalled gastrulation as a result of $N v A x 6$ knockdown is, in part, a result of the upregulation and expansion observed by the NvAx1 transcript, which antagonizes oral development (Fig. $3 \mathrm{~m}$ ). To test this hypothesis, we co-injected both NvAx6 (oral) and $N v A x 1$ (aboral) morpholinos into $N$. vectensis zygotes and assessed changes in late gastrulae (48hpf). Controls were injected with either $N v A x 6$ or $N v A x 1$ morpholinos with a standard control morpholino and produced phenotypes identical to those seen with normal single gene-specific morpholino injections (Fig. 6). Embryos treated with both $N v A x 6$ and $N v A x 1$ morpholinos undergo gastrulation to form an outer ectoderm (Ec) and an inner endomesoderm (En) that expresses the maker $N v$ SnailA, but fail to form a pharynx $(\mathrm{P})$ or express the pharyngeal markers NvFoxA and NvBrachyury (Fig. 6). Aboral markers assessed in this study were lost in double injection $(N v A x 6+$ $N v A x 1)$ experiments, including a complete loss of $N v \operatorname{Six} 3 / 6$ (Fig. 6), which is reduced but not lost in $N v A x 1$ morpholino treatments. Co-injection of $N v A x 6$ and $N v A x 1$ morpholinos has no effect on oral marker expression, including the pharyngeal marker NvFoxA at earlier gastrula stages (24hpf) (Supplementary Fig. 3), suggesting that patterning cues downstream of the oral Hox gene, $N v A x 6$, are required after the onset of gastrulation. However, the aboral marker $N v \operatorname{Six} 3 / 6$ is lost at $24 \mathrm{hpf}$ in treated embryos (Supplementary Fig. 3), demonstrating that $N v A x 1$ expression has a patterning role at these earlier gastrula stages.

$N v A x 6$ and $N v A x 1$ regulate oral-aboral axis through $W n t$. The oral-aboral axis of cnidarians is thought to be patterned by restricted Wnt domains beginning at the onset of gastrulation and progressing through larval development ${ }^{38-43}$. NvWnt1, NvWnt3, $N v W n t 4$, and $N v W n t A$ are expressed around the blastopore/oral 


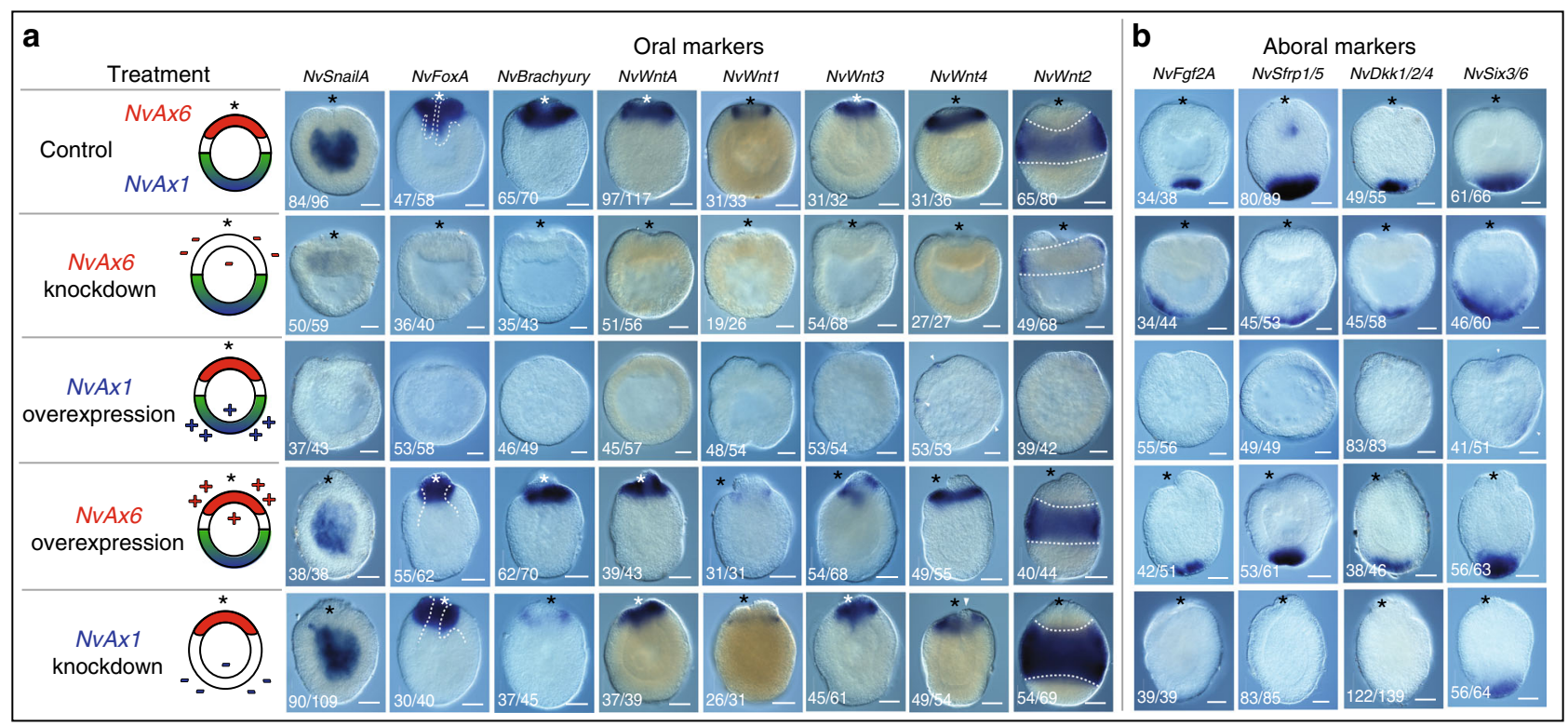

C

Gene expression domain

NvFoxA
NvWnt3
NvBrachyury, NvWntA, NvWnt1, NvWnt4
NvWnt2
NvSnailA
NvSix3/6
NvFgt2A, NvSfrp 1/5, NvDkk1/2/4

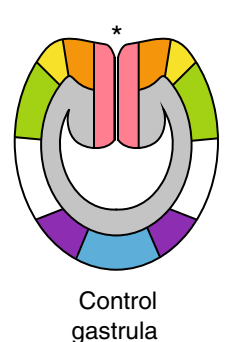

gastrula

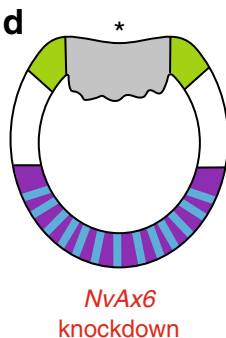

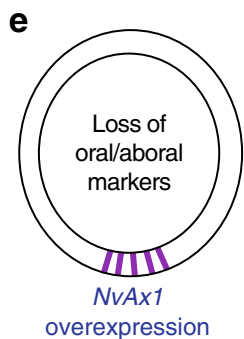
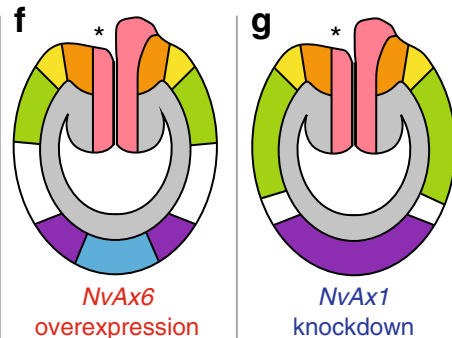

Fig. 5 Anterior and central/posterior Hox genes have reciprocal phenotypes during oral but not aboral development. a Schematic representation of perturbation of either anterior Hox $(\mathrm{NvAx} 6)$ or central/posterior Hox (Ax1) during early development. a, b Late gastrula (48hpf) stage expression of molecular markers for oral (a) or aboral (b) territories. White-dashed lines in NvFoxA column serve to highlight the larval pharynx and distinguish between the ectoderm and endoderm. White-dashed lines in the NvWnt2 column outline the belt of NvWnt2 expression. c-g Illustration summarizing territorial changes due to manipulation of anterior ( NvAx6) and central/posterior (NvAx1) Hox genes. Images were compiled from at least three separate experiments and the number of similar phenotypes is noted as a fraction in the lower left-hand corner. Scale bars are $50 \mu \mathrm{m}$

pole and disappear following both oral Hox NvAx6 knockdown and aboral Hox NvAx1 overexpression (Fig. 5a). NvWnt2 is expressed in a band along the ectodermal midline of the embryo and serves as an important marker for defining oral and aboral territories. Knockdown of Hox NvAx6 expression results in shift of $N v W n t 2$ expression toward the oral pole, while overexpression of $N v A x 6$ has no observable effect on $N v W n t 2$ expression (Fig. 5a). NvWnt2 expression is lost in aboral Hox (NvAx1) overexpression treatments, while knockdown of $N v A x 1$ resulted in a robust upregulation and aboral expansion of the $N v W n t 2$ domain (Fig. 5a). A more pronounced phenotype was produced in CRISPR cas9-mediated knockout of $N v A x 1$, resulting in upregulation of $N v W n t 2$ throughout the entire ectoderm and endomesoderm (Supplementary Fig. 4). NvAx1 knockouts also expanded the orally expressed $N v W n t 1$ into the endomesoderm (Supplementary Fig. 4). These findings suggest that $N v W n t 2$ is the intermediary between the ectodermal oral-aboral Hox gene boundary and that territorial Wnt expression is mediated by blastula Hox patterning.

\section{Discussion}

Since the first cnidarian genome became available $e^{44}$ it has become clear that much of the developmental "toolkit" deployed during the development of bilaterian species remains present in the cnidarian lineage. Although there are aspects of cnidarian Hox clusters that have diverged from the ancestral condition in virtually all lineages (e.g., loss or duplication of Hox2 homologs, diversification of central/posterior genes in Hydra, Supplementary Fig. 1), it appears that the common ancestor of cnidarians and bilaterians utilized Hox genes to pattern their primary (oral-aboral) body axis. If a Hox cluster is representative of the ancestral condition in Cnidaria, gene duplications and loss may have played an instrumental role in the divergent life history evolution (e.g., metagenic lifecyles) within the phylum. For example, the hydrozoan cnidarian, Clytia hemisphaerica, has a medusa (jellyfish) life history stage and very similar set of Hox genes with Nematostella ${ }^{21}$. However, comparison of the ancestral role of Hox genes in these two species are difficult to make at this time because the genomic organization of Hox genes in Clytia is unresolved, and similar to early work in Nematostella ${ }^{15,20}$, expression analysis by in situ hybridization may only have highlighted stages exhibiting elevated levels of mRNA. Additionally, no experiments have investigated the embryonic patterning role of Hox genes in any other cnidarian. Previous studies in Hydra, which lacks both the larva and medusa lifecycle stages ${ }^{45,46}$, has highlighted the role of Hox genes in patterning the adult polyp oral-aboral axis ${ }^{31,33,34}$. It will be interesting to see if gene loss (e.g., loss of Eve in Hydra) and cluster fragmentation helped shape the diverse life history stages and embryonic development of cnidarians.

There exists a longstanding argument about the origin of axial patterning machinery outside of bilaterians. Our results indicate 


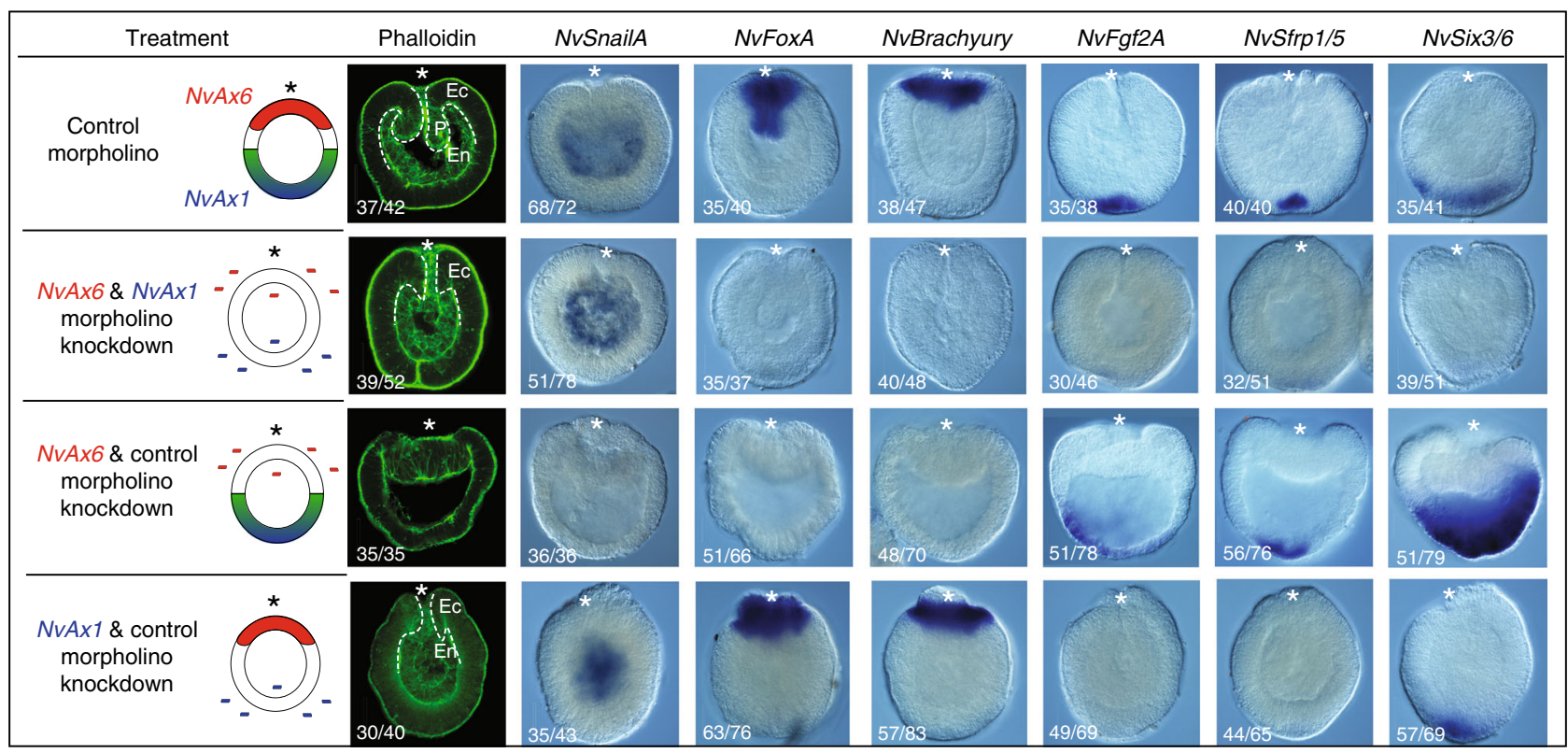

Fig. 6 Loss of anterior and central/posterior Hox results in loss of oral-aboral patterning, but not endomesoderm formation. Gastrulation defects resulting from co-injection experiments as assessed by fluorescent phalloidin labeling (far left column) and in situ hybridization for oral (NvSnailA, NvBrachyury, and NvFoxA) and aboral (NvFgf2a, NvSfrp1/5, and NvSix3/6) markers. Ec ectoderm; En endo-mesodermal; and P pharynx. White-dashed line serves to distinguish the point of transition between the ectoderm and the endoderm. Images were compiled from at least three separate experiments and the number of similar phenotypes is noted as a fraction in the lower left-hand corner

that anterior and central/posterior Hox genes play important roles specifying oral and aboral territories, respectively, prior to the onset of gastrulation. During gastrulation and on through planula development, a complex developmental program is initiated that involves $\mathrm{Wnt} / \beta$-catenin signaling that patterns the oral-aboral axis. During this time, Bmp agonists/antagonists activate Hox genes in the endomesoderm layer along a second axis, perpendicular to the oral-aboral axis, called the directive axis (may or may not be homologous to the dorsal-ventral axis of bilaterians) ${ }^{47-49}$. The directive axis is likely established around the onset of gastrulation, after establishment of the oral-aboral axis at the site of gastrulation. If the evolutionary origins of distinct clades of Hox genes (e.g, anterior class Hox genes) pattern similar regions of cnidarian body plans, as they do in bilaterians (i.e., anterior body regions), our findings would argue that the oral pole is homologous to the bilaterian anterior pole and the posterior end would be equivalent to the cnidarian aboral pole. This is consistent with numerous studies that show the cnidarian animal pole gives rise to the oral pole ${ }^{50-54}$, just as it does in virtually all other bilaterians ${ }^{5-58}$ and in another early branching basal animal lineage, the ctenophores ${ }^{59,60}$. If the phylogenetic relationship of these genes also coincides with axial positioning, these findings would suggest that the oral-aboral axis is homologous to the bilaterian A-P axis, with the adult mouth corresponding to anterior in both cases. The alternatives are that A-P positioning never existed prior to bilaterians ${ }^{13}$ or it could be argued that early animals may have possessed only "anterior-like" Hox genes without true central/posterior class genes ${ }^{14}$. The later scenario appears to be less likely, as medusazoan cnidarians like Clytia and Hydra appear to have true central/posterior class genes $^{21}$, although greater taxonomic sampling is needed. Despite what we know about Hox genes in cnidarians, some components of the molecular patterning of the apical tuft neurosensory cilia region appear to be similar in different invertebrate larvae ${ }^{61-63}$. Further characterization of this transient larval apical tuft region across cnidarians is warranted, because some of the most robust indicators like the homeobox gene, Six $3 / 6$, is expressed orally in other cnidarians ${ }^{64}$. Of course, additional studies are required to determine with certainty the molecular basis for axial development in animal evolution.

In this report, we have characterized for the first time in any cnidarian embryo the existence of early domains of reciprocal Hox gene expression. In the starlet sea anemone, Nematostella vectensis, an anterior Hox gene is expressed at the beginning of blastula stages, extending through gastrulation and functionally interacts with a maternally expressed central/posterior Hox gene in oral-aboral axis formation and tissue specification. Although suggested to be primarily a vertebrate phenomenon ${ }^{65}$, elements of the hierarchal prevalence among Hox genes appears to be functioning in Nematostella. NvAx6 and NvAx1 act in a partial antagonistic fashion to pattern both oral and aboral territories (Fig. 7a, b), which appears to be mediated through interactions with Wnt signaling. Through these studies, we can begin to understand the molecular basis of oral-aboral patterning in Nematostella and develop a working model of cnidarian Hox function (Fig. 7c).

During early cleavage stages, the stabilization of $\beta$-catenin and components of both canonical and PCP Wnt signaling pathways establish the site of gastrulation/future oral pole in Nematostella $^{66,67}$ (Fig. 7c; I). Recent findings have shown that $N v \beta$-catenin activity is upstream of NvBrachyury ${ }^{50}$ and that NvBrachyury is upstream of Wnt signaling ${ }^{51}$ at the oral pole, a process that is partially mediated by the homeobox gene NvSix3/6 ${ }^{68}$ (Fig. 7c; II). Our findings suggest that the maternal aboral marker NvAx1 also suppresses activation of Wnt signaling at the oral pole, when overexpressed, potentially through blocking activation of $N v A x 6$ and downstream targets (e.g., $N v B r a, N v W n t A, N v W n t 1, N W n t 3$, and $N v$ Wnt4) (Fig. $7 \mathrm{c}$; IV). These results are consistent with crosstalk between $N v \beta$-catenin and aboral patterning ${ }^{68}$, where oral $N v \beta$-catenin is necessary to initiate aboral patterning. Therefore, the loss of aboral genes as a result of NvAxl overexpression may be a result of the inactivation of oral $\beta$-catenin, in turn effecting both oral and aboral specification. Recent work began to establish the gene regulatory network driving 


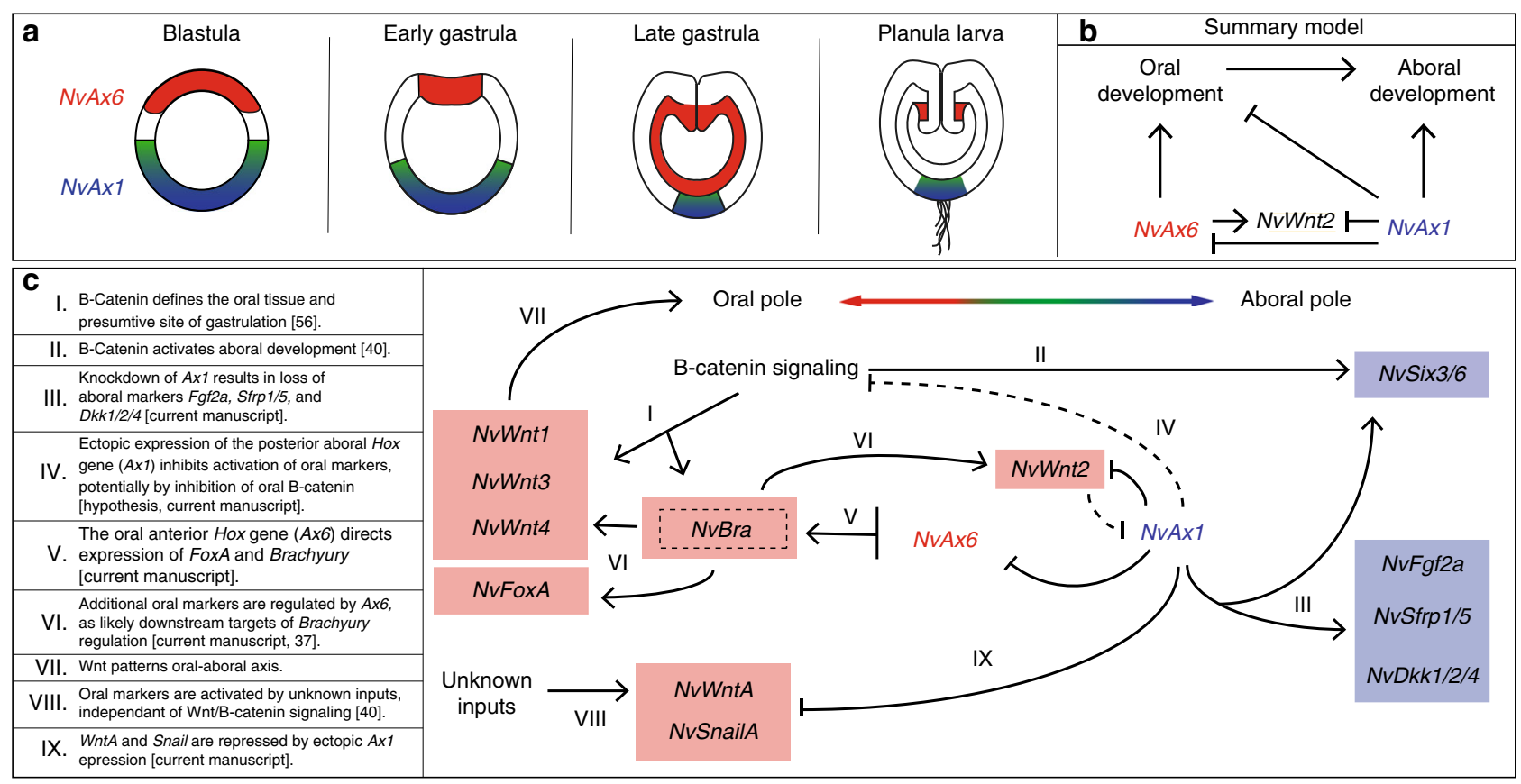

Fig. 7 Model of Hox gene function in early development of an anthozoan. a lllustration depicting anterior Hox (NvAx6) (red) and central/posterior Hox (NvAx1) (blue/green) expression throughout early development of $N$. vectensis. b Working model of how anterior (NvAx6) and central/posterior ( NvAx1) Hox genes function to specify the oral-aboral axis before and during gastrulation in an anthozoan cnidarian. c Illustration of known regulatory relationships between Hox genes and the oral (NvWntA, NvWnt1, NvWnt2, NvWnt3, NvWnt4, NvFoxA, NvBrachyury, and NvSnailA) and aboral (NvSix3/6, NvFgf2A, NvSfrp1/5, and NvDkk1/2/4) markers assessed in this study. Important components of this regulatory paradigm needing description are marked with roman numerals $(I-I X)$ with corresponding text at the periphery of the illustration. Regulatory relationships derived from previous studies are cited in the periphery text $(\mathrm{I}, \mathrm{II}, \mathrm{VI}$, and VIII)

endomesoderm formation in Nematostella, and highlighted the blastopore ring domains along the oral/animal pole ${ }^{35}$. Our findings add greater detail to this network and suggest that the NvWnt2 expression domain could be the boundary region between dedicated oral-aboral territories. Hyper activation of $\beta$ catenin signaling through pharmacological treatment was shown to shift $N v W n t 2$ boundaries toward the aboral pole, while suppressing $N v A x 1^{43}$. Nv $\beta$-catenin knockdown abolishes $N v$ Wnt2 expression $^{68}$ yet the functional link with oral and aboral Hox genes has yet to be resolved.

The formation of drastically elongated polyps in aboral Hox (NvAx1) knockdown (Fig. 4l) points to a captivating similarity between the functions of vertebrate and anthozoan central/posterior (and posterior-like) Hox genes in axial elongation. Similar phenotypes were also witnessed as a result of mis-regulation of $N v \beta$-catenin signaling ${ }^{66}$, providing greater evidence for a link between these two molecular pathways. Results from the double knockdown of $N v A x 6$ and $N v A x 1$ provides support for the proposed role of $N v A x 1$ in restricting oral specification, possibly through the restriction of Wnt signaling, as parallel knockdown of NvAx1 was sufficient to rescue the loss of gastrulation and oral specification phenotype produced in NvAx6 morpholino treatments. From these results, we propose that $N v A x 1$ is an inhibitor of $\mathrm{Wnt} / \beta$-catenin signaling (Fig. $7 \mathrm{c} ; \mathrm{IV}$ ) similar to what has recently been described during vertebrate development ${ }^{69}$. Therefore, the loss of $N v A x 1$ expression in oral territories is necessary for establishment and progression of oral patterning and the core regulatory relationship between central/posterior (posterior-like) Hox expression and Wnt signaling may be a highly conserved characteristic of posterior Hox function. Further investigation into the mechanism of this relationship and the hierarchal nature of the other NvHox genes will help determine if the paradigm of posterior dominance is truly conserved between bilaterians and cnidarians, and will help resolve if $N v A x 6$ exhibits any form of anterior dominance.

The phylogenetic origin of the Hox patterning system is a highly controversial matter among evolutionary developmental biologists. The cnidarian clade has the greatest diversity of Hox genes among the four basal animal lineages (sponge and ctenophore genomes do not contain Hox genes $\left.{ }^{25,26,28}\right)$. Genomic resources from a diverse group of cnidarians suggest rearrangements and fragmentations of genomic clusters are present among cnidarian species. The axial patterning role of cnidarian $\mathrm{Hox}$ genes was long debated $21,31-34$, yet the functional analysis of Hox genes in Nematostella indicate that some aspects of genomic organization and A-P patterning were present in the cnidarian ancestor. It will be interesting if the study of other cnidarian species reveal an additional role for Hox genes during early development, which will ultimately help resolve the shared and derived characters of cnidarian and bilaterian Hox genes. Together these data suggest that cnidarians retain components of a simple ancestral Hox system that was originally deployed during early developmental stages and functioned to organize the primary axis in the cnidarian ancestor.

\section{Methods}

Animal care. Adult Nematostella vectensis were raised in $1 / 3 \times$ seawater at $16^{\circ} \mathrm{C}$ in constant dark. Animals were fed artemia once a week and fed oyster $48 \mathrm{~h}$ before spawning, which was engendered by an 8 -h light box cycle at $16^{\circ} \mathrm{C}$. Distinct groups of animals were spawned once every 3-4 weeks. Fertilized embryos were collected and placed in a $4 \%$ cysteine wash ( $4 \%$ cysteine in $1 / 3$ filtered seawater, $\mathrm{pH} 7.4$ ) for 15 min to remove the outer jelly layer. Eggs were then washed $(3 \times)$ with $1 / 3$ filtered seawater before conducting experiments. Treated embryos were raised to collection at $16^{\circ} \mathrm{C}$. Embryos used were from randomized individuals from different genetic individuals to eliminate genetic variability. Mixed genetic pools of individual animals created a blind test of the effectiveness of each treatment regardless of genetic ancestry. 
Microinjection knockdown overexpression and knockout. We disrupted $N v A x 6$ and $N v A x 1$ Hox gene expression using gene-specific antisense translation blocking morpholinos or CRISPR/Cas9-mediated genome editing using multiple guide RNAs (gRNAs) against each gene ${ }^{36,49}$. Genes were over expressed by injecting in vitro transcribed mRNA created using mMessage in vitro transcription kits (Ambion AM1348). Morphological and molecular analysis of phenotypes was conducted during late gastrula (48hpf), planula larvae (96hpf) stages of development, and 1-2-week-old polyps. Control injections using a standard control morpholino and dextran lineage tracer (or Cas9 protein without gRNAs in knockout injections) produced normal gastrula stage embryos (Fig. 3k) possessing an outer ectoderm(Ec), inner endomesoderm(En), and pharynx(P) (dashed white line) (Supplementary Fig. 2). Knockdown and knockout treatments produced similar molecular phenotypes (Supplementary Fig. 4), thus validating the efficacy of the $N v A x 6$ and $N v A x 1$ morpholinos. Treated embryos were raised to collection at $16^{\circ} \mathrm{C}$.

Translation blocking morpholinos against $N v A x 6$ and $N v A x 1$ and a standard control morpholino have been developed through Gene Tools, LLC. Philomath OR, 97370. Morpholino sequences are as follows (Standard Control 5'-CCTCTTACCT CAGTTACAATTTATA-3'; NvAntHox6 5'-ACCGCCGCTCATGCCCAAA TGTGTC-3'; NvAntHox1 5'-TTGACTGCATGATGTGCGCTCTAGT-3'). Expression constructs for $N v A x 6$ and $N v A x 1$ were generated using the gateway cloning system (SPE3-Ax1-Rvenus, SPE3-Ax6-RmCherry, and SPE3-Ax6aRmCherry). Morpholinos and mRNA were first injected in a dilution series ranging in concentration from 0.1 and $0.9 \mathrm{mM}$ and appropriate concentrations $(0.3 \mathrm{mM}$ for NvAx6 MO, $0.5 \mathrm{mM} N v A x 6 \mathrm{mRNA}, 0.9 \mathrm{mM}$ for $N v A x 1$, and $0.3 \mathrm{mM} N v A x 1$ mRNA) were selected to achieve an optimal relationship between toxicity and phenotype penetrance.

CRISPR/Cas9 genome editing in Nematostella has recently become a useful tool for genetic manipulation ${ }^{36,49}$. To perform knockouts of Hox genes, target sequences $18-20 \mathrm{bp}$ in length fitting the gRNA target profile of $5^{\prime}-\mathrm{G}(\mathrm{G}-\mathrm{A})-\mathrm{N}(16-$ 18)-NGG-3' were identified within the coding sequence of the $N v A x 6$ and $N v A x 1$ locus (Supplementary Figs 5a, 6a) using a web-based program called ZiFiT (http:// zifit.partners.org). Six target sequences were selected for each locus bases on their location (choosing sites scattered throughout the coding sequence) and their gRNA efficiency score, which was calculated using the web-based CRISPR Efficiency Predictor provided by the DRSC at Harvard Medical School (http://www.flyrnai. org/evaluateCrispr/). Sequences and efficiency scores are listed in Supplementary Table 1. Additionally, potential target sequences were blasted against the publically available Nematostella genome (http://genome.jgi.doe.gov) to insure that there were minimal off-target sites related to the sequence, limiting partial off-target sites to having no more than 15 base pairs in common with the 18-20 base pair target sequence. Oligonucleotides were generated integrating the target sequence ( $5^{\prime}-\mathrm{G}$ (G-A)-N(16-18)-NGG-3') into a CRISPR RNA (crRNA) sequence containing a T7 (5'- AATTAATACGACTCACTATA-3') or Sp6 (5'-AATATTTAGGTGACA CTATA- ${ }^{\prime}$ ) promoter. Full-length gRNA was generated from a PCR assembled trans-activating crRNA (tracrRNA) oligonucleotide (Supplementary Table 1), followed by in vitro transcription (NEB Highscribe ${ }^{\text {me }}$ T7 and Sp6 RNA synthesis kits; Cat\# E2040S and E2070S), and RNA purification (Zymogen RNA Clean and Concentrator ${ }^{\mathrm{nt}}-25$; Cat\# R1017). gRNAs at a final concentration of $400 \mathrm{ng} / \mu \mathrm{l}$ (consisting of equal concentrations of each gRNA) were injected with Lyophilized bacterial type II Cas9 protein (PNA Bio, Thousand Oaks, CA) $(1 \mu \mathrm{g} / \mu \mathrm{l})$ reconstituted in $50 \%$ glycerol and $0.1 \mathrm{mM}$ DTT to generate frame shift mutations and deletions within the target locus. Genomic DNA was isolated from 8 individual $18 \mathrm{hpf}$ embryos per treatment group for PCR analysis of the targeted region using a DNA extraction kit, according to the manufacturers protocol (Qiagen 69504) (Supplementary Figs 5b, 6b). Additionally, in situ hybridization was performed on treated blastula stage embryos to ensure that gene expression was lost

(Supplementary Figs 5c, 6c). Treated embryos were collected at $48 \mathrm{hpf}$ for in situ hybridization studies to assess changes in marker gene expression (Supplementary Fig. 4).

Quantitative PCR. Quantitative PCR was performed using the Roche LightCycler ${ }^{\circ}$ 480 Instrument II and LightCycler 480 SYBR Green I Master mix (Cat\# 04707516001, Roche, Inc.). qPCR samples were standardized with $N v G A D P H$ and NvRiboPro and primers for other genes were designed using MacVector (www. macvector.com) to amplify 75-150 base-pair fragments of the desired gene. These primers were then back-blasted against the Nematostella genome to make sure they only will amplify a single region from the genome. We checked each primer efficiency with a dilution curve $\left(10^{-1}-10^{-5}\right)$ to make sure their range was within the negligible value of 1.9-2.0. Individual stages were collected from pooled samples of embryos consisting of roughly 100 embryos. These stages represent a single biological sample, and were confirmed through technical replication. Total RNA from each sample was stored in TRIzol (15596-026) at $-80^{\circ} \mathrm{C}$ until processed. RNA was isolated according to manufacturer's specification. RNA was DNase treated using Qiagen RNase-free DNase \#79254 for $15 \mathrm{~min}$ at $37^{\circ} \mathrm{C}$. cDNA was standardized by using $1 \mu \mathrm{g}$ of total RNA and synthesis was conducted using the Advantage RT-PCR kit (Clontech, 639506) for qPCR analysis. Samples were run using a Roche LightCycler 480 qPCR platform. Roche SYBR Green 1 Master mix (Roche 04887352001) was used for all qPCR. Relative fold change values were calculated in Microsoft Excel.
In situ hybridization. All in situ hybridizations were conducted as described below. Fixations were done in 1\% gelatin-coated dishes to prevent tissue from sticking to the plastic (sticking to plastic causes tissue damage and non-specific staining). Embryos were fixed in ice cold $4 \%$ paraformaldehyde with $0.2 \%$ glutaraldehyde in $1 / 3 \times$ seawater for $2 \mathrm{~min}$, followed by $4 \%$ paraformaldehyde in $1 / 3 \times$ seawater for $1 \mathrm{~h}$ at $4{ }^{\circ} \mathrm{C}$. Embryos were stored in $100 \%$ methanol at $-20^{\circ} \mathrm{C}$ until used. Samples were rehydrated through a methanol series $(75 \%$ methanol/25\% PBS $+0.1 \%$ Tween $20,50 \%$ methanol $/ 50 \%$ PBS $+0.1 \%$ Tween $20,25 \%$ methanol $/ 75 \%$ PBS $+0.1 \%$ Tween 20$)$ and then washed $3 \times$ in PBS $+0.1 \%$ Tween $20(\mathrm{Ptw})$. Embryos were treated with $0.1 \mathrm{mg} / \mathrm{ml}$ Proteinase $\mathrm{K}$ in Ptw for $5 \mathrm{~min}$, followed by $2 \times$ washes in Ptw $+2 \mathrm{mg} / \mathrm{ml}$ glycine. Embryos were moved to 24 -well tissue culture plates, then incubated in $0.1 \%$ triethanolamine in Ptw for 5 min, followed by two washes with $0.1 \%$ triethanolamine in $\mathrm{Ptw}+3 \mu \mathrm{l} / \mathrm{ml}$ of acetic anhydride. Samples were then washed $2 \times$ in Ptw, followed by a $1 \mathrm{~h}$ fixation in $4 \%$ PFA in Ptw at room temperature. Afterward samples were washed $5 \times$ in Ptw and then incubated overnight in hybridization buffer.

Probe sequences ranging from 550-1200 bps were cloned from cDNA using the pGEM $^{\oplus}-\mathrm{T}$ vector system and DIG-labeled RNA probes were generated using MEGAscript in vitro transcription kits (Invitrogen, AM1333M). If available, cloned marker gene sequences were selected from a probe stock originally created for a previous study ${ }^{35}$. Probes were hybridized at $64^{\circ} \mathrm{C}$ for 2 days and developed with the enzymatic reaction of NBT/BCIP as substrate for the alkaline phosphataseconjugated anti-DIG antibody (Cat\# 11093274910, Roche, Inc.). Wild-type samples were developed for an equal amount of time and if no expression was visible, a subset of samples remained in developing solution for at least 1 day to determine if lower levels of expression was present. When developing in situs of treated embryos, development time was based on the signal seen in control samples. All experiments exhibited greater than $50 \%$ penetrance of phenotypes, with $75 \%$ of the data containing greater than $75 \%$ penetrance. Due to variability during injection, embryo size and development time of different genes, greater than $50 \%$ in replicates, were considered the dominant phenotype created by genetic manipulations. Furthermore, to obtain material to test all the genes, samples were collected from multiple days of injection and pooled for in situ hybridization. Replicate attempts of in situ hybridization were attempted at least twice on pooled samples to obtain sample sizes generally greater than 30 embryos per replicate.

Immunostaining. Methods for immunostaining were adapted from previously published protocols ${ }^{51}$. Embryos collected for immunostaining were fixed in ice cold $4 \%$ paraformaldehyde with $0.2 \%$ glutaraldehyde in $1 / 3 \times$ seawater for $2 \mathrm{~min}$, followed by $4 \%$ paraformaldehyde in $1 / 3 \times$ seawater for $1 \mathrm{~h}$ at $4{ }^{\circ} \mathrm{C}$. Samples were then washed five times ( 5 min each) in PBT (1\% Triton-x and $1 \%$ BSA in PSS) and stored in PBS at $4{ }^{\circ} \mathrm{C}$ for up to 1 month. Samples were then washed three times (30 min each) in PBT followed by a 1-h block in blocking solution (5\% normal goat serum in PBT) at room temperature. Samples were then treated with the primary antibody (monoclonal anti- $\alpha$-Tubulin; SigmaT9026) diluted 1:500 in blocking solution and incubated overnight $(12 \mathrm{~h})$ at $4{ }^{\circ} \mathrm{C}$. Primary antibody was removed and samples were rinsed five times $(10 \mathrm{~min}$ each) in PBT before adding the secondary antibody (goat-anti-mouse-568; A-11004) diluted 1:250 in blocking solution and incubated over night at $4{ }^{\circ} \mathrm{C}$. Tissues were then washed at least three times $(15 \mathrm{~min}$ each) in PBT before adding counterstain. DAPI was used $(0.1 \mu \mathrm{g} / \mu \mathrm{l}$ in PBS) was used to label cell nuclei. Fluorescent phalloidin (Invitrogen A12379) was used at a concentration of 1:200 in PBT to stain filamentous actin, incubating for no more than $3 \mathrm{~h}$ at room temperature. Samples were washed at least three times (15 min each) in PBS before clearing. Samples were cleared in $80 \%$ glycerol or using Murray's Clear (1:1 benzyl benzoate and benzyl alcohol).

Microscopy and imaging. Results from in situ hybridization studies were imaged using a Zeiss Axio Imager Z1 with a Zeiss HRc color digital camera run by Zeiss Zen 2012 software. Fluorescently labeled embryos were imaged using a Zeiss LSM710 confocal microscope. Images of polyp stage results and whole wells of 24-well plates (as pictured in Fig. 4 and Supplementary Figs 5-6) were imaged using A Zeiss SteREO Discovery.V8 stereoscopic microscope with a Cannon EOS 5D MarkII full frame DSLR camera. Images were processed and scale bars were added using the Fiji distribution of ImageJ (http://fiji.sc).

Data availability. The authors declare that all data supporting the findings of this study are available within the article and its Supplementary Information files or from the corresponding author upon reasonable request. All figures and supplementary data are available through FigShare (https://doi.org/10.6084/m9. figshare.5900887). All primers used for generation of gRNA for CRISPR-Cas9 are available in Supplementary Table 1. Full-length transcripts used for probe production, mRNA misexpression, and designing morpholinos are available in the Supplementary Table 1. All protocols can be requested through Nature Protocol Exchange under the Martindale Whitney Lab group.

Received: 21 September 2017 Accepted: 6 April 2018 Published online: 22 May 2018 


\section{References}

1. Lewis, E. B. A gene complex controlling segmentation in Drosophila. Nature 276, 565-570 (1978).

2. Akam, M. Hox and HOM: homologous gene clusters in insects and vertebrates. Cell 57, 347-349 (1989).

3. Lemons, D. \& McGinnis, W. Genomic evolution of Hox gene clusters. Science 313, 1918-1922 (2006)

4. Boncinelli, E. et al. Organization of human homeobox genes. Hum. Reprod. 3, 880-886 (1988).

5. Duboule, D. \& Dollé, P. The structural and functional organization of the murine HOX gene family resembles that of Drosophila homeotic genes. EMBO J. 8, 1497-1505 (1989).

6. Kaufman, T., Seeger, M. \& Olsen, G. Molecular and genetic organization of the Antennapedia gene complex of Drosophila melanogaster. Adv. Genet. 27, 309-362 (1990).

7. Gaunt, S. J. \& Strachan, L. Temporal colinearity in expression of anterior hox genes in developing chick embryos. Dev. Dyn. 207, 270-280 (1996).

8. Amores, A. et al. Developmental roles of pufferfish Hox clusters and genome evolution in ray-fin fish. Genome Res. 14, 1-10 (2004).

9. Iimura, T. \& Pourquié, O. Collinear activation of Hoxb genes during gastrulation is linked to mesoderm cell ingression. Nature 442, 568-571 (2006).

10. Fröbius, A. C., Matus, D. Q. \& Seaver, E. C. Genomic organization and expression demonstrate spatial and temporal Hox gene colinearity in the lophotrochozoan Capitella sp. I. PLoS ONE 3, e4004 (2008).

11. Schiemann, S. M. et al. Clustered brachiopod Hox genes are not expressed collinearly and are associated with lophotrochozoan novelties. Proc. Natl Acad. Sci. USA 114, E1913-E1922 (2017).

12. Wagner, G. P., Amemiya, C. \& Ruddle, F. Hox cluster duplications and the opportunity for evolutionary novelties. Proc. Natl Acad. Sci. USA 100, 14603-14606 (2003).

13. Kamm, K., Schierwater, B., Jakob, W., Dellaporta, S. L. \& Miller, D. J. Axial patterning and diversification in the cnidaria predate the Hox system. Curr. Biol. 16, 920-926 (2006).

14. Chourrout, D. et al. Minimal ProtoHox cluster inferred from bilaterian and cnidarian Hox complements. Nature 442, 684-687 (2006).

15. Ryan, J. F. et al. Pre-bilaterian origins of the hox cluster and the hox code: evidence from the sea anemone, Nematostella vectensis. PLoS ONE 2, e153 (2007).

16. Mendivil, R. O., Barker, D. \& Ferrier, D. E. K. Ghost loci imply Hox and ParaHox existence in the last common ancestor of animals. Curr. Biol. 22, 1951-1956 (2012).

17. Simakov, O. et al. Insights into bilaterian evolution from three spiralian genomes. Nature 493, 526-531 (2013).

18. Pascual-Anaya, J., D’Aniello, S., Kuratani, S. \& Garcia-Fernàndez, J. Evolution of Hox gene clusters in deuterostomes. BMC Dev. Biol. 13, 26 (2013).

19. Hejnol, A. \& Martindale, M. Q. Coordinated spatial and temporal expression of Hox genes during embryogenesis in the acoel Convolutriloba longifissura. BMC Biol. 7, 65 (2009).

20. Finnerty, J. R., Pang, K., Burton, P., Paulson, D. \& Martindale, M. Q. Origins of bilateral symmetry: Hox and dpp expression in a sea anemone. Science 304, 1335-1337 (2004)

21. Chiori, R. et al. Are Hox genes ancestrally involved in axial patterning? Evidence from the hydrozoan Clytia hemisphaerica (Cnidaria). PLoS ONE 4, e4231 (2009).

22. DuBuc, T. Q., Ryan, J. F., Shinzato, C., Satoh, N. \& Martindale, M. Q. Coral comparative genomics reveal expanded Hox cluster in the cnidarian-bilaterian ancestor. Integr. Comp. Biol. 52, 835-841 (2012).

23. Baumgarten, S. et al. The genome of Aiptasia, a sea anemone model for coral symbiosis. Proc. Natl Acad. Sci. USA 112, 11893-11898 (2015).

24. Bourlat, S. J. \& Hejnol, A. Acoels. Curr. Biol. 19, R279-R280 (2009).

25. Ryan, J. F. et al. The genome of the ctenophore Mnemiopsis leidyi and its implications for cell type evolution. Science 342, 1242592 (2013).

26. Moroz, L. L. et al. The ctenophore genome and the evolutionary origins of neural systems. Nature 510, 109-114 (2014).

27. Leininger, S. et al. Developmental gene expression provides clues to relationships between sponge and eumetazoan body plans. Nat. Commun. 5, 3905 (2014).

28. Srivastava, M. et al. The Amphimedon queenslandica genome and the evolution of animal complexity. Nature 466, 720-726 (2010).

29. McGinnis, W. \& Krumlauf, R. Homeobox genes and axial patterning. Cell 68, 283-302 (1992).

30. Durston, A. J. in Embryogenesis (ed. Sato, K.-I.) 131-150 (IntechOpen, 2012).

31. Gauchat, D. et al. Evolution of Antp-class genes and differential expression of Hydra Hox/paraHox genes in anterior patterning. Proc. Natl Acad. Sci. USA 97, 4493-4498 (2000).

32. Jakob, W. \& Schierwater, B. Changing hydrozoan bauplans by silencing Hoxlike genes. PLoS ONE 2, e694 (2007).
33. Schummer, M., Scheurlen, I., Schaller, C. \& Galliot, B. HOM/HOX homeobox genes are present in hydra (Chlorohydra viridissima) and are differentially expressed during regeneration. EMBO J. 11, 1815-1823 (1992).

34. Bode, H. R. The role of Hox genes in axial patterning in Hydra. Am. Zool. 41, 621-628 (2001).

35. Röttinger, E., Dahlin, P. \& Martindale, M. Q. A framework for the establishment of a cnidarian gene regulatory network for 'endomesoderm' specification: the inputs of ß-catenin/TCF signaling. PLoS Genet. 8, e1003164 (2012).

36. Servetnick, M. et al. Cas9-mediated excision of Nematostella brachyury disrupts endoderm development, pharynx formation and oral-aboral patterning. Development 144, 2951-2960 (2017).

37. Yasuoka, Y., Shinzato, C. \& Satoh, N. The mesoderm-forming gene brachyury regulates ectoderm-endoderm demarcation in the coral Acropora digitifera. Curr. Biol. 26, 2885-2892 (2016).

38. Hobmayer, B. et al. WNT signalling molecules act in axis formation in the diploblastic metazoan Hydra. Nature 407, 186-189 (2000).

39. Kusserow, A. et al. Unexpected complexity of the Wnt gene family in a sea anemone. Nature 433, 156-160 (2005).

40. Lee, P. N., Pang, K., Matus, D. Q. \& Martindale, M. Q. A WNT of things to come: evolution of Wnt signaling and polarity in cnidarians. Semin. Cell Dev. Biol. 17, 157-167 (2006).

41. Guder, C. et al. The Wnt code: cnidarians signal the way. Oncogene 25, 7450-7460 (2006)

42. Trevino, M., Stefanik, D. J., Rodriguez, R., Harmon, S. \& Burton, P. M. Induction of canonical Wnt signaling by alsterpaullone is sufficient for oral tissue fate during regeneration and embryogenesis in Nematostella vectensis. Dev. Dyn. 240, 2673-2679 (2011)

43. Marlow, H., Matus, D. Q. \& Martindale, M. Q. Ectopic activation of the canonical wnt signaling pathway affects ectodermal patterning along the primary axis during larval development in the anthozoan Nematostella vectensis. Dev. Biol. 380, 324-334 (2013).

44. Putnam, N. H. et al. Sea anemone genome reveals ancestral eumetazoan gene repertoire and genomic organization. Science 317, 86-94 (2007).

45. Martin, J., Archer, E., Littlefield, C. L. \& Bode, H. R. Embryogenesis in Hydra. Biol. Bull. 192, 345-363 (1997).

46. Nawrocki, A. M., Collins, A. G., Hirano, Y. M., Schuchert, P. \& Cartwright, P. Phylogenetic placement of hydra and relationships within Aplanulata (Cnidaria: Hydrozoa). Mol. Phylogenet. Evol. 67, 60-71 (2013).

47. Matus, D. Q., Thomsen, G. H. \& Martindale, M. Q. Dorso/ventral genes are asymmetrically expressed and involved in germ-layer demarcation during cnidarian gastrulation. Curr. Biol. 16, 499-505 (2006).

48. Saina, M. \& Technau, U. Characterization of myostatin/gdf8/11 in the starlet sea anemone Nematostella vectensis. J. Exp. Zool. B Mol. Dev. Evol. 312, 780-788 (2009).

49. Wijesena, N., Simmons, D. K. \& Martindale, M. Q. Antagonistic BMP-cWNT signaling in the cnidarian Nematostella vectensis reveals insight into the evolution of mesoderm. Proc. Natl Acad. Sci. USA 114, E5608-E5615 (2017).

50. Freeman, G. The role of polarity in the development of the hydrozoan planula larva. Wilhelm. Roux's Arch. Dev. Biol. 190, 168-184 (1981).

51. Freeman, G. Experimental studies on embryogenesis in hydrozoans (Trachylina and Siphonophora) with direct development. Biol. Bull. 165, 591-618 (1983)

52. Momose, T. \& Schmid, V. Animal pole determinants define oral-aboral axis polarity and endodermal cell-fate in hydrozoan jellyfish Podocoryne carnea. Dev. Biol. 292, 371-380 (2006).

53. Fritzenwanker, J. H., Genikhovich, G., Kraus, Y. \& Technau, U. Early development and axis specification in the sea anemone Nematostella vectensis. Dev. Biol. 310, 264-279 (2007).

54. Lee, P. N., Kumburegama, S., Marlow, H. Q., Martindale, M. Q. \& Wikramanayake, A. H. Asymmetric developmental potential along the animal-vegetal axis in the anthozoan cnidarian, Nematostella vectensis, is mediated by Dishevelled. Dev. Biol. 310, 169-186 (2007).

55. Cameron, R., Hough-Evans, B., Britten, R. \& Davidson, E. Lineage and fate of each blastomere of the 8-cell sea-urchin embryo. Genes Dev. 1, 75-84 (1987).

56. Dale, L. \& Slack, J. M. W. Fate map for the 32-cell stage of Xenopus laevis. Development 99, 527-551 (1987).

57. Gardner, R. L. The early blastocyst is bilaterally symmetrical and its axis of symmetry is aligned with the animal-vegetal axis of the zygote in the mouse. Development 124, 289-301 (1997)

58. Henry, J. Q., Tagawa, K. \& Martindale, M. Q. Deuterostome evolution: early development in the enteropneust hemichordate, Ptychodera flava. Evol. Dev. 3, 375-390 (2001)

59. Freeman, G. The establishment of the oral-aboral axis in the ctenophore embryo. J. Embryol. Exp. Morphol. 42, 237-260 (1977).

60. Martindale, M. Q. \& Henry, J. Q. Intracellular fate mapping in a basal metazoan, the ctenophore Mnemiopsis leidyi, reveals the origins of mesoderm and the existence of indeterminate cell lineages. Dev. Biol. 214, 243-257 (1999).

61. Sinigaglia, $\mathrm{C}$. et al. The bilaterian head patterning gene six $3 / 6$ controls aboral domain development in a cnidarian. PLoS Biol. 11, e1001488 (2013). 
62. Marlow, $\mathrm{H}$. et al. Larval body patterning and apical organs are conserved in animal evolution. BMC Biol. 12, 7 (2014).

63. Arendt, D., Tosches, M. A. \& Marlow, H. From nerve net to nerve ring, nerve cord and brain-evolution of the nervous system. Nat. Rev. Neurosci. 17, 61-72 (2016).

64. Kraus, J. E. M., Fredman, D., Wang, W., Khalturin, K. \& Technau, U. Adoption of conserved developmental genes in development and origin of the medusa body plan. EvoDevo 6, 23 (2015).

65. Durston, A. J. Global posterior prevalence is unique to vertebrates: a dance to the music of time? Dev. Dyn. 241, 1799-1807 (2012).

66. Wikramanayake, A. H. et al. An ancient role for nuclear beta-catenin in the evolution of axial polarity and germ layer segregation. Nature 426, 446-450 (2003).

67. Wijesena, N. M., Kumburegama, S., Xu, R. \& Wikramanayake, A. Wnt signaling in the cnidarian Nematostella vectensis: insights into the evolution of gastrulation. Dev. Biol. 356, 107 (2011).

68. Leclère, L., Bause, M., Sinigaglia, C., Steger, J. \& Rentzsch, F. Development of the aboral domain in Nematostella requires $\beta$-catenin and the opposing activities of six3/6 and frizzled5/8. Development 1, 1766-1777 (2016).

69. Denans, N., Iimura, T. \& Pourquié, O. Hox genes control vertebrate body elongation by collinear Wnt repression. eLife 4, 04379 (2015).

70. Shippy, T. D. et al. Analysis of the Tribolium homeotic complex: insights into mechanisms constraining insect Hox clusters. Dev. Genes Evol. 218, 127-139 (2008).

\section{Acknowledgements}

The authors would like to thank Dr. Joseph Ryan for help with evolutionary orthology assignments, Dr. Elaine Seaver for help with Fig. 1, and Dr. Marten Postma for advice with qPCR analysis and Fig. 2. We would also like to acknowledge the National Science Foundation (NSF) for continuous funding of this project under three different grants: "Broadening participation of underrepresented groups in Developmental Biology" (NSFIOS-1239422), "Research Experience for Undergraduates" (NSF-DBI-1156528), and NSF-IOS-1338420, and the National Institute of Health (NIH) for their funding that helped support this project (NIH GM093116).

\section{Author contributions}

T.Q.D., T.B.S., and M.Q.M. performed all microinjections. T.Q.D., T.B.S., and A.Q.R. performed phenotype analysis and in situ hybridization experiments. T.Q.D. performed all qPCR and T.B.S. conducted CRISPR-Cas9 experiments. All four authors contributed to the design of experiments and generation of the manuscript.

\section{Additional information}

Supplementary Information accompanies this paper at https://doi.org/10.1038/s41467018-04184-x.

Competing interests: The authors declare no competing interests.

Reprints and permission information is available online at http://npg.nature.com/ reprintsandpermissions/

Publisher's note: Springer Nature remains neutral with regard to jurisdictional claims in published maps and institutional affiliations.

\section{(c) (i)}

Open Access This article is licensed under a Creative Commons Attribution 4.0 International License, which permits use, sharing, adaptation, distribution and reproduction in any medium or format, as long as you give appropriate credit to the original author(s) and the source, provide a link to the Creative Commons license, and indicate if changes were made. The images or other third party material in this article are included in the article's Creative Commons license, unless indicated otherwise in a credit line to the material. If material is not included in the article's Creative Commons license and your intended use is not permitted by statutory regulation or exceeds the permitted use, you will need to obtain permission directly from the copyright holder. To view a copy of this license, visit http://creativecommons.org/ licenses/by/4.0/.

(C) The Author(s) 2018 